\title{
Integration Roadmap for Multi-Scale, Multi-Physics Mass Accountancy in Molten Salt Reactors
}

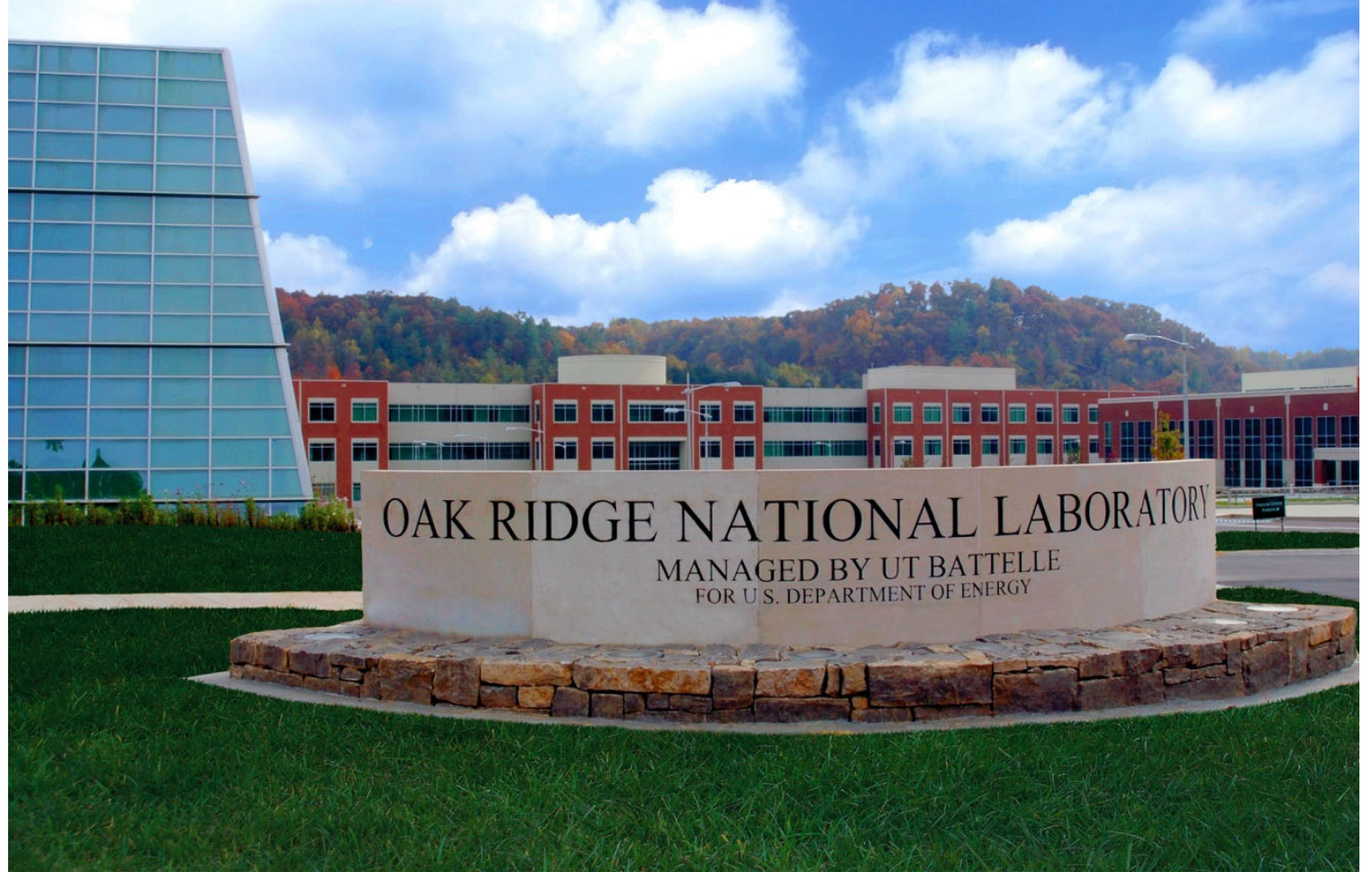
J McMurray
K Johnson
B Collins
A Graham
K Lee
B Betzler
Z Taylor
B Salko
$S$ Henderson
M Piro
$\mathrm{R} \mathrm{Hu}$
D Holcomb

September 2021 


\title{
DOCUMENT AVAILABILITY
}

Reports produced after January 1, 1996, are generally available free via US Department of Energy (DOE) SciTech Connect.

Website www.osti.gov

Reports produced before January 1, 1996, may be purchased by members of the public from the following source:

\author{
National Technical Information Service \\ 5285 Port Royal Road \\ Springfield, VA 22161 \\ Telephone 703-605-6000 (1-800-553-6847) \\ TDD 703-487-4639 \\ Fax 703-605-6900 \\ E-mail info@ntis.gov \\ Website http://classic.ntis.gov/
}

Reports are available to DOE employees, DOE contractors, Energy Technology Data Exchange representatives, and International Nuclear Information System representatives from the following source:

Office of Scientific and Technical Information

PO Box 62

Oak Ridge, TN 37831

Telephone 865-576-8401

Fax 865-576-5728

E-mail reports@osti.gov

Website https://www.osti.gov/

This report was prepared as an account of work sponsored by an agency of the United States Government. Neither the United States Government nor any agency thereof, nor any of their employees, makes any warranty, express or implied, or assumes any legal liability or responsibility for the accuracy, completeness, or usefulness of any information, apparatus, product, or process disclosed, or represents that its use would not infringe privately owned rights. Reference herein to any specific commercial product, process, or service by trade name, trademark, manufacturer, or otherwise, does not necessarily constitute or imply its endorsement, recommendation, or favoring by the United States Government or any agency thereof. The views and opinions of authors expressed herein do not necessarily state or reflect those of the United States Government or any agency thereof. 
Nuclear Energy Advanced Modeling Simulation Program

INTEGRATION ROADMAP FOR MULTI-SCALE, MULTI-PHYSICS MASS ACCOUNTANCY IN MOLTEN SALT REACTORS

\author{
Author(s) \\ J McMurray \\ K Johnson \\ B Collins \\ A Graham \\ K Lee \\ B Betzler \\ Z Taylor \\ B Salko \\ S Henderson \\ M Piro \\ R Hu \\ D Holcomb
}

September 2021

Prepared by

OAK RIDGE NATIONAL LABORATORY

Oak Ridge, TN 37831-6283

managed by

UT-BATTELLE LLC

for the 
US DEPARTMENT OF ENERGY

under contract DE-AC05-00OR22725 



\section{CONTENTS}

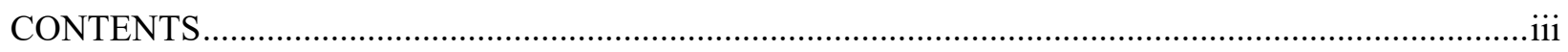

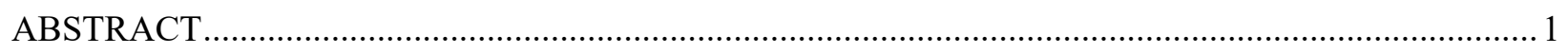

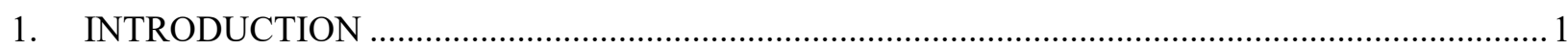

2. SPECIES IDENTIFICATION FOR SELECTIVE TRACKING ......................................................2

2.1 CHEMISTRY AND DOSE CONSEQUENCE …........................................................ 3

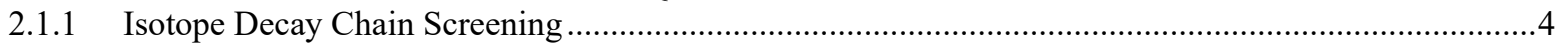

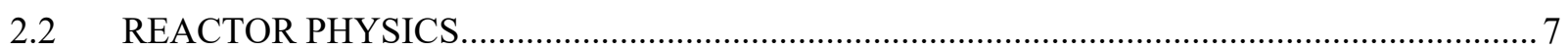

3. THE MOLTEN SALT THERMAL PROPERTIES DATABASE (MSTDB) .................................. 10

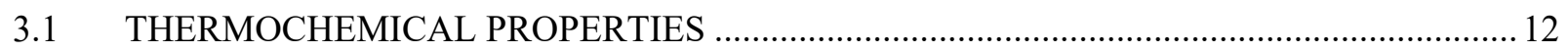

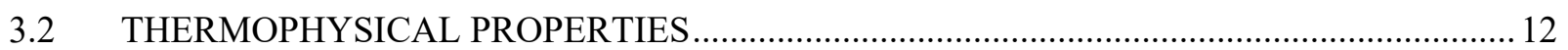

3.3 COMPUTATIONALLY DERIVED THERMAL PROPERTIES ............................................. 12

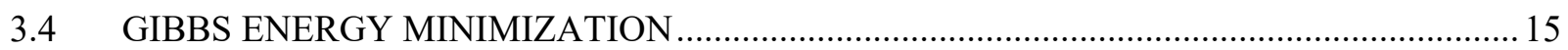

3.5 SILANE APPLICATION PROGRAMMING INTERFACE …................................................ 15

4. MODELING AND SIMULATION OF MASS ACCOUNTANCY ….............................................. 16

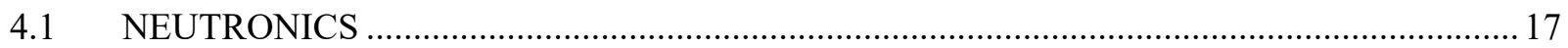

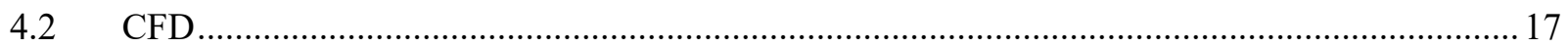

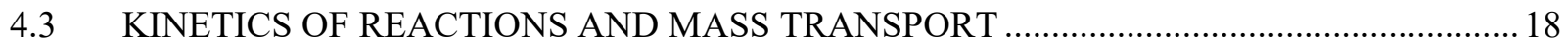

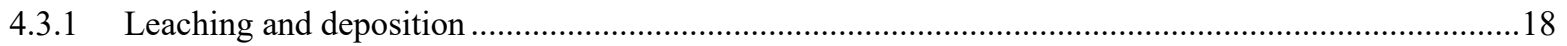

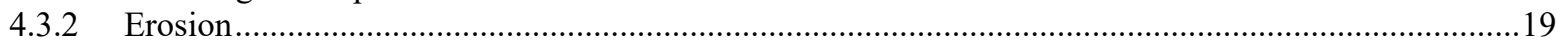

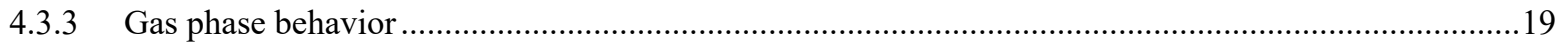

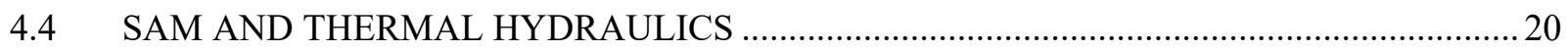

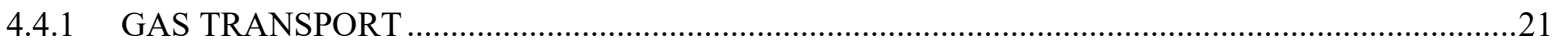

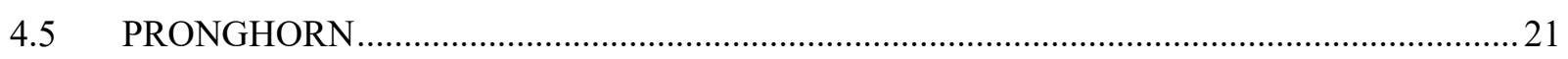

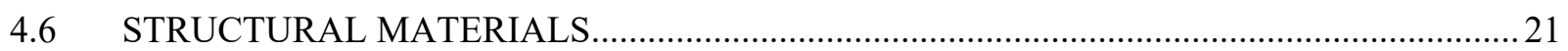

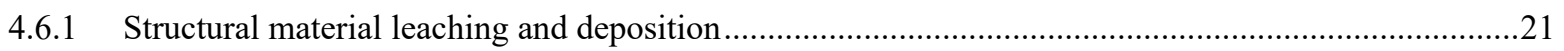

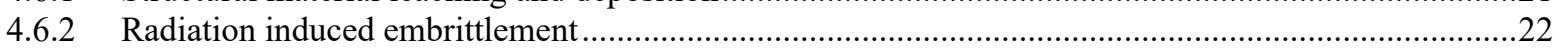

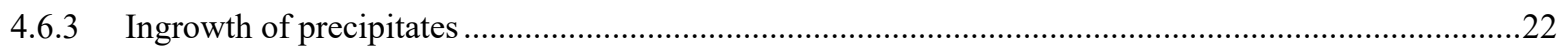

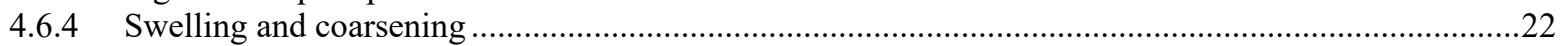

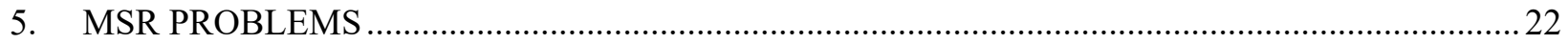

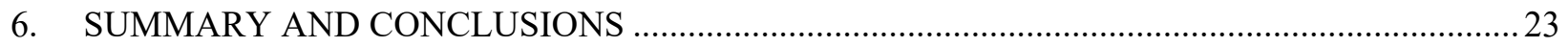

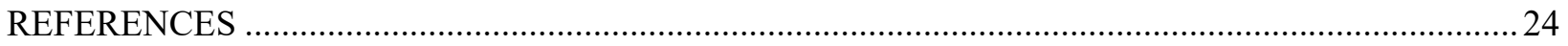

Appendix A. burnup simulation results ……................................................................................ 3 


\begin{abstract}
The objective of the integration roadmap is to describe the necessary physics required and a plan for modeling and simulation approach for predicting mass accountancy in molten salt reactors on an engineering scale. The thermophysical properties and the underlying thermodynamics are fundamental inputs. Therefore, the modeling will span length scales from first principles calculations to the engineering scale. The intention is to predict where material accumulates in a reactor core and loop and to understand perturbations on the systems level, for example the downstream effects from a turbine failure.
\end{abstract}

\title{
1. Introduction
}

The US Department of Energy Nuclear Energy (DOE) Advanced Modeling and Simulation (NEAMS) program aims to develop codes for supporting industry efforts to design and operate advanced reactors, for example those using a molten salt, whether fueled or not. The Nuclear Regulatory Commission (NRC) relies upon computational tools like MELCOR [1] to evaluate the radionuclide release potential of accident scenarios. Codes for addressing safety adequacy of Molten Salt Reactors (MSRs) require further development. The DOE Molten Salt Reactor (MSR) campaign supports advancing MSR technology by developing technical information and tools for the NRC and industry and performing data and methodology validation experiments under appropriate quality control that are responsive to the data needs of those stakeholders. Modeling and simulation and experimentation are inextricably linked. A utilitarian paradigm is as follows. First, NEAMS tools are used to rapidly and cost effectively generate property estimates that are subsequently validated by targeted measurements performed within the MSR campaign.

The overall need for MSR Modeling and Simulation (M\&S) in terms of safety adequacy assessment is to develop conservative estimates of the quantities, species, and forms of radioactive materials that may be released from MSRs as the result of accidents. The estimates cannot, however, be so unrealistically conservative to significantly impact overall plant costs. Thus, the degree of fidelity of the models, data, and simulations needs to be sufficient to support the needs of both the plant owner/operator and the safety regulator.

A typical approach to assessing the required precision of the fuel salt property as well as M\&S fidelity would be to:

1. Develop mechanistic plant modeling tools for both normal conditions and accidents.

2. Generate fuel salt property data, under an appropriate quality assurance program, over a sufficient range of normal and accident conditions and that includes experimental error bounds.

3. Develop a set of design basis accidents for the plant under evaluation using any acceptable method

4. Validate the modeling tools - The modeling of each specific plant safety feature (including potential interdependent effects) would be validated through a combination of analysis, testing, and experience over a range of conditions that includes normal operations, transient conditions, and design basis accident sequences.

5. Evaluate the amount of radionuclide release for each design basis accident sequence using the validated modeling tools and conservative estimates of the fuel salt properties derived from the measured data.

6. For any accident sequence for which the $M \& S$ indicates the potential for an unacceptable amount of radionuclide release, evaluate the technical basis for the release estimate. 
7. As necessary, improve the safety characteristics of the design, increase the fidelity of the model, and/or decrease the uncertainty in the fuel salt property data until no accident sequence results in unacceptable releases.

The behavior of an MSR is driven to a large degree by both nuclear and chemical phenomena. Mass accountancy, that is the action of determining the chemical state, physical characteristics, and timedependent location of species in a system, is necessary to address the questions needed to design and operate MSRs as well as aid regulators in making licensing decisions. The principle phenomena are leaching, deposition, and vapor phase transport of important radionuclides. The NEAMS program is developing mass accountancy modeling tools for MSRs spanning length scales, incorporating fundamental thermodynamic driving forces, and coupling to thermal hydraulics and reactor physics.

Development of the NEAMS Yellowjacket Corrosion Suite was initiated in FY20. Progress was made for computationally derived thermal properties (density, viscosity, thermal conductivity, and specific heat) using classical and ab-initio molecular dynamics. Yellowjacket-PF is a mesoscale phase field [2] [3] code. It is a tool for simulating diffusion processes, microstructural evolution, and pore formation using thermodynamic inputs, specifically chemical potentials and phase equilibria. Mole is a code for solving species transport problems on the engineering scale. This includes a wide variety of phenomena, for example advection, leaching from system components into a fluid, phase transformations like deposition of species from a fluid onto system components or gas bubble formation, diffusion of species between gas bubbles and the fluid, etc. The code is built to be flexible, allowing for a wide range of different species to be simulated over a wide variety of time scales. While not a part of the Yellowjacket Corrosion Suite proper, the thermal hydraulics of the loop is simulated with the Systems Analysis Module (SAM), a plant transient analysis code for fast turnaround design scoping and safety analyses of advanced non-light-water reactors [4] [5]. The Yellowjacket-GEM is a Gibbs Energy Minimizer (GEM) for coupling thermodynamics to multi-physics, multi-scale codes in MOOSE [3]. It is intended to support modeling and simulation relevant to nuclear fuel systems, i.e. molten salts, oxides, nitrides, alloys etc. The GEM is needed to utilize the thermodynamic part of the Molten Salt Thermal Properties Database, or MSTDB-TC.

The MOOSE framework will be used to couple codes from the Yellowjacket suite that primarily treats corrosion, to those for reactor physics, mass transport, thermal hydraulics, and structural mechanics [6]. Information is passed between codes, modules, submodules, etc. For example, kinetics of solid-state diffusion of corrosion products are an output of Yellowjacket-PF. This information is provided for boundary conditions in the MOSCATO (Molten Salt Chemistry and Transport) computational fluid dynamics (CFD) code. Coupling MOSCATO to the engineering scale results from reduced order models informs the treatment of mass transport around a molten salt system in Mole and SAM. Sink terms from MOLE and SAM are provided to Yellowjacket-PF for material balancing. Mole, SAM, MOSCATO, and Yellowjacket-PF all need thermal property inputs that must be taken from experimental measurements and/or derived from first principles calculations. Finally, yellowjacket-PF is the coupling point between the chemistry and continuum mechanics codes by providing compositional profiles and microstructure, both of which dominate structural material behavior. The SAM code provides velocity and temperature fields needed for mass transport (e.g. surface plating, erosion, advection) and phase transformations (e.g. precipitation and dissolution) that can be modeled with Mole.

\section{Species Identification for Selective Tracking}

Tracking isotopes that impact (1) chemistry (2) reactor physics and (3) dose consequence will be important for modeling and simulation of mass accountancy in MSRs. Preliminary burnup calculations 
using the MSRE [7] and the $\mathrm{SOFT}^{1}$ [8] reactor designs were used to gain an understanding of the isotopes that are generally expected to be present in both a chloride and fluoride MSR. A depletion data set was generated with the SCALE/TRITON continuous processing capability [9], which are derived from the ChemTriton tool [10] [11]. Parameters for the simulation of the two designs are given in Table 1 and Table 2. The results are tabulated in the Appendix.

Table 1 Parameters used for simulating the isotopic inventory for the thermal neutron spectrum fluoride carrier salt MSRE reactor.

\begin{tabular}{l|l}
\hline Fuel type & $35 \%$ enriched $\mathrm{U}^{235}$ \\
\hline Carrier salt (mole \%) & $69.1 \mathrm{LiF}-30.9 \mathrm{BeF}_{2}$ \\
\hline Fuel chemical form & $\mathrm{UF}_{4}$ \\
\hline Temperature $\left({ }^{\circ} \mathbf{C}\right)$ & 649 \\
\hline Salt Mixture $\left(\mathbf{m o l e}^{\mathbf{\%})}\right.$ & $65 \mathrm{LiF}-29.1 \mathrm{BeF} 2-5 \mathrm{ZrF}_{4}-0.9 \mathrm{UF}_{4}$ \\
\hline Salt volume $\left(\mathbf{m}^{3}\right)$ & 2.07 \\
\hline Fueling condition/rate & $\mathrm{NA}$ \\
\hline
\end{tabular}

Table 2 Parameters used for simulating the elemental inventory for the fast neutron spectrum chloride carrier salt SOFT reactor.

\begin{tabular}{l|l}
\hline Fuel type & $\mathrm{U} / \mathrm{Pu}$ \\
\hline Carrier salt & $\mathrm{NaCl}$ \\
\hline Fuel chemical form & $\mathrm{PuCl}_{3}, \mathrm{UCl}_{3}$ \\
\hline Temperature $\left({ }^{\circ} \mathbf{C}\right)$ & 537 \\
\hline Salt Mixture $(\mathbf{m o l e} \%)$ & $1 \mathrm{PuCl}_{3}-8 \mathrm{UCl}_{3}-10 \mathrm{NaCl}$ \\
\hline Salt volume $\left(\mathbf{m}^{3}\right)$ & 107 \\
\hline Fueling condition/rate & $\mathrm{Load}$ depleted $\mathrm{U}$ to maintain overall salt density \\
\hline
\end{tabular}

\subsection{CHEMISTRY AND DOSE CONSEQUENCE}

The major fission products are categorized in Table 3. From a mass accountancy perspective, any isotope that transitions between phases must be tracked. These were identified using comprehensive decay chains from available nuclear structure and decay data. ${ }^{2}$ Iodine and Cs are included in the analysis because of the importance to dose consequence.

Table 3 Categories of major fission products. The soluble fission products (salt seekers) are those that are stable as halides and are expected to show some degree of solubility in the base molten salt.

\begin{tabular}{ccc}
\hline Salt seekers & Noble gases & Noble metals \\
$\mathrm{RE}$ 's $(\mathrm{Nd}, \mathrm{Ce}, \mathrm{La}, \mathrm{Pr})$ & $\mathrm{Xe}$ & $\mathrm{Ru}$ \\
$\mathrm{Zr}$ & $\mathrm{Kr}$ & $\mathrm{Pd}$ \\
$\mathrm{Cs}$ & $\mathrm{He}$ & $\mathrm{Rh}$ \\
\hline
\end{tabular}

\footnotetext{
${ }^{1}$ The SOFT reactor design was a thought experiment for a specific purpose - to minimize the amount of circulating radionuclides. This makes it particularly unrealistic for radionuclide accountancy. The DOE NE fuel cycle campaign has investigated two other chloride salt fast reactors - MCSFR (UKAEA) and REBUS-3700. It is recommended that this work and the companion Roadmap for thermal property measurements of Molten Salt Reactor systems be updated in FY22 to reflect a more realistic fast spectrum chloride molten salt reactor design.

${ }^{2}$ International Atomic Energy Agency, "LiveChart of Nuclides," 2021. https://wwwnds.iaea.org/relnsd/vcharthtml/VChartHTML.html.
} 


\begin{tabular}{cc}
\hline $\mathrm{Ba}$ & $\mathrm{Mo}^{*}$ \\
$\mathrm{Sr}$ & $\mathrm{Tc}^{*}$ \\
$\mathrm{Pu}$ & \\
$\mathrm{Mo}^{*}$ & \\
$\mathrm{Tc}^{*}$ & \\
\hline
\end{tabular}

\subsubsection{Isotope Decay Chain Screening}

Each individual stream (fuel, gas and solid waste) from the depletion calculation discussed above was considered independently. To a first order, the impact of an element on the overall chemical behavior of the salt loop will scale with compositional abundance. Therefore, in addition to Cs and I, only the isotopes present in concentrations greater than $0.0001 \mathrm{~mol} \%$ are considered. The daughters and parents of the most abundant radioisotopes were screened to identify cases that involved either a transition involving a noble gas, I, or between salt seeker (potential soluble halide-forming species) and insoluble isotopes. Some examples are shown in Table 4. Products of decays with half-life of $10^{5}$ years or longer were considered the stable isotopes. For salts with high Li loading, tritium generation is a concern but can likely be tracked separately with simple analytical approaches [12].

Table 4. Screening decision examples.

\begin{tabular}{|c|c|c|c|}
\hline Isotope & Decay Chain & Screening & Criteria \\
\hline Ba-138 & ${ }^{138} \mathrm{Xe} \stackrel{14 m}{\longrightarrow}{ }^{138} \mathrm{Cs} \stackrel{33 m}{\longrightarrow}{ }^{138} \mathrm{Ba}$ & Included & Decay of a noble gas into a salt seeker. \\
\hline $\mathrm{Nb}-95$ & ${ }^{95} \mathrm{Nb} \stackrel{35 d}{\longrightarrow}{ }^{95} \mathrm{Mo}$ & Excluded & $\begin{array}{l}\text { Decay to isotopes that do not change from noble } \\
\text { (insoluble) behavior }\end{array}$ \\
\hline Rh-103 & ${ }^{103} \mathrm{Tc} \stackrel{54 s^{s}}{\longrightarrow}{ }^{103} R u \stackrel{39 d}{\longrightarrow}{ }^{103} \boldsymbol{R} \boldsymbol{h}$ & Excluded & $\begin{array}{l}\text { Rapid decay from a likely noble element to } \\
\text { noble daughters }\end{array}$ \\
\hline Y-91 & ${ }^{91} \stackrel{Y^{59 d}}{\longrightarrow}{ }^{91} \mathrm{Zr}$ & Excluded & $\begin{array}{l}\text { Decay to isotopes that do not change from salt } \\
\text { seeker behavior }\end{array}$ \\
\hline $\mathrm{I}-129$ & ${ }^{129} \mathrm{Sb} \stackrel{24 h^{129}}{\longrightarrow} \mathrm{Te} \stackrel{70 m}{\longrightarrow}{ }^{129} \boldsymbol{I}$ & Included & $\begin{array}{l}\text { Presence of I relevant to public health and dose } \\
\text { consequence }\end{array}$ \\
\hline I-129 & ${ }^{129} I \stackrel{10^{7} y}{\longrightarrow}{ }^{129} \mathrm{Xe}$ & Excluded & $\begin{array}{l}\text { Appreciable accumulation of Xe-129 will not } \\
\text { occur in relevant timeframe }\end{array}$ \\
\hline Cs-137 & ${ }^{137} I \stackrel{25 s}{\longrightarrow}{ }^{137} \mathrm{Xe} \stackrel{4 m}{\longrightarrow}{ }^{137} \mathrm{Cs} \stackrel{30 y}{\longrightarrow}{ }^{137} \mathrm{Ba}$ & Included & $\begin{array}{l}\text { Decay of a volatile and noble gas into salt } \\
\text { seeker. }\end{array}$ \\
\hline
\end{tabular}

MSRE - Fuel

Half-life: $m=$ minutes, $d=$ days, $y=$ years

\begin{tabular}{|c|c|c|c|}
\hline \multicolumn{3}{|c|}{ Concentration (mol\%) } & \multirow[b]{2}{*}{ Associated Decay Chain } \\
\hline Isotope & Year 1 & Year 30 & \\
\hline Ba-138 & 0.0003 & 0.0087 & ${ }^{138} \mathrm{Xe} \stackrel{14 m}{\longrightarrow}{ }^{138} \mathrm{Cs} \stackrel{33 m}{\longrightarrow}{ }^{138} \mathrm{Ba}$ \\
\hline Cs-137 & 0.0003 & 0.0059 & ${ }^{137} \mathrm{I} \stackrel{25 s}{\longrightarrow}{ }^{137} \mathrm{Xe} \stackrel{4 \mathrm{~m}}{\rightarrow}{ }^{137} \mathrm{Cs} \stackrel{30 y_{1}}{\longrightarrow}{ }^{137} \mathrm{Ba}$ \\
\hline Y-89 & 0.0002 & 0.0053 & ${ }^{89} \mathrm{Zr} \stackrel{78 h}{\rightarrow}{ }^{89} \boldsymbol{Y} \quad$ or $\quad{ }^{89} \mathrm{Sr} \stackrel{51 d}{\longrightarrow}{ }^{89} \boldsymbol{Y}$ \\
\hline $\mathrm{Rb}-85$ & 0.0000 & 0.0011 & ${ }^{85} \mathrm{Br} \stackrel{3 m^{m}}{\longrightarrow}{ }^{85} \mathrm{Kr} \stackrel{11 y^{85}}{\longrightarrow} \boldsymbol{R} \boldsymbol{b}$ \\
\hline $\mathrm{I}-127$ & 0.0000 & 0.0001 & ${ }^{127} \mathrm{Cs} \stackrel{6 h}{\rightarrow}{ }^{127} \mathrm{Xe} \stackrel{36 d}{\longrightarrow}{ }^{127} \boldsymbol{I}$ \\
\hline Cs-135 & 0.0000 & 0.0001 & ${ }^{135} I \stackrel{7 h}{\rightarrow}{ }^{135} \mathrm{Xe} \stackrel{9 h^{135}}{\rightarrow} \mathrm{Cs}$ \\
\hline \multicolumn{4}{|c|}{ MSRE - Gas Waste } \\
\hline \multicolumn{3}{|c|}{ Concentration (mol\%) } & Associated Decay Chain \\
\hline
\end{tabular}




\begin{tabular}{|c|c|c|c|}
\hline $\mathrm{Xe}-134$ & 22.1566 & 21.4608 & ${ }^{134} \mathrm{I} \stackrel{83 s}{\longrightarrow}{ }^{134} \mathrm{Xe} \quad$ or $\quad{ }^{134} \mathrm{Cs} \stackrel{2 y}{\rightarrow}{ }^{134} \mathrm{Xe}$ \\
\hline Cs-135 & 18.3714 & 18.6738 & ${ }^{135} I \stackrel{7 h}{\rightarrow}{ }^{135} \mathrm{Xe} \stackrel{9 h}{\rightarrow}{ }^{135} \mathrm{Cs}$ \\
\hline Cs-133 & 18.4315 & 18.3749 & ${ }^{133} I I^{21 h}{ }^{133} \mathrm{Xe} \stackrel{5 d}{\rightarrow}{ }^{133} \mathrm{Cs}$ \\
\hline $\mathrm{Xe}-136$ & 17.8487 & 17.8815 & ${ }^{136} I \stackrel{83 s}{\longrightarrow}{ }^{136} \mathrm{Xe}$ \\
\hline $\mathrm{Xe}-131$ & 7.9973 & 8.1421 & ${ }^{131} \stackrel{8 d}{\rightarrow}{ }^{131} \mathrm{Xe} \quad$ or $\quad{ }^{131} \mathrm{Cs} \quad \stackrel{10 d}{\longrightarrow}{ }^{131} \mathrm{Xe}$ \\
\hline $\mathrm{Kr}-86$ & 5.4808 & 4.6755 & ${ }^{86} \mathrm{Br} \stackrel{55 s}{\longrightarrow}{ }^{86} \mathrm{Kr} \quad$ or $\quad{ }^{87} \mathrm{Br} \stackrel{56 s}{\longrightarrow}{ }^{86} \mathrm{Kr} \quad$ or ${ }^{86} \mathrm{Rb} \stackrel{19 d}{\longrightarrow}^{86} \mathrm{Kr}$ \\
\hline $\mathrm{Kr}-84$ & 2.8039 & 2.4344 & ${ }^{84} \mathrm{Br} \stackrel{32 m}{\longrightarrow}{ }^{84} \mathrm{Kr} \quad$ or $\quad{ }^{84} \mathrm{Rb} \stackrel{33 d}{\longrightarrow}{ }^{84} \mathbf{K r}$ \\
\hline $\mathrm{Xe}-132$ & 1.7317 & 1.8384 & ${ }^{132} I \stackrel{2 h}{\rightarrow}{ }^{132} \mathrm{Xe} \quad$ or $\quad{ }^{132} \mathrm{Cs} \stackrel{6 d}{\rightarrow}{ }^{132} \mathrm{Xe}$ \\
\hline $\mathrm{Kr}-83$ & 1.5001 & 1.3193 & ${ }^{83} \mathrm{Br} \stackrel{2 h}{\rightarrow}{ }^{83} \mathrm{Kr} \quad$ or $\quad{ }^{83} \mathrm{Rb} \stackrel{86 d}{\longrightarrow}{ }^{83} \mathbf{K r}$ \\
\hline $\mathrm{Rb}-85$ & 0.0238 & 0.4014 & ${ }^{85} \mathrm{Br} \stackrel{3 m}{\longrightarrow}{ }^{85} \mathrm{Kr} \stackrel{11 y}{\rightarrow}{ }^{85} \boldsymbol{R} \boldsymbol{b}$ \\
\hline $\mathrm{Kr}-85$ & 0.7713 & 0.2852 & ${ }^{85} \mathrm{Kr} \stackrel{11 y^{85}}{\longrightarrow} R b$ \\
\hline $\mathrm{Xe}-130$ & 0.0022 & 0.1997 & ${ }^{130} I \stackrel{12 h}{\longrightarrow}{ }^{130} \mathrm{Xe} \quad$ or $\quad{ }^{130} \mathrm{Cs} \stackrel{{ }^{29 m}}{\longrightarrow}{ }^{130} \mathrm{Xe}$ \\
\hline $\mathrm{Xe}-128$ & 0.0004 & 0.0219 & ${ }^{128} I \stackrel{25 m}{\longrightarrow}{ }^{128} X e$ \\
\hline Kr-82 & 0.0005 & 0.0160 & ${ }^{82} \mathrm{Br} \stackrel{35 h}{\rightarrow}{ }^{82} \mathbf{K r} \quad$ or $\quad{ }^{82} R b \stackrel{1 m}{\longrightarrow}{ }^{82} \mathbf{K r}$ \\
\hline Xe-133 & 0.4131 & 0.0118 & ${ }^{133} I \stackrel{21 h}{\longrightarrow}{ }^{133} \boldsymbol{X e} \stackrel{5 d}{\rightarrow}{ }^{133} \mathrm{Cs}$ \\
\hline $\mathrm{Xe}-135$ & 0.0288 & 0.0008 & ${ }^{135} I \stackrel{7 h}{\rightarrow}{ }^{135} \boldsymbol{X e} \stackrel{9 h}{\rightarrow}{ }^{135} \mathrm{Cs}$ \\
\hline
\end{tabular}


MSRE - Solid Waste

\begin{tabular}{|c|c|c|c|}
\hline \multicolumn{4}{|c|}{ Concentration (mol\%) } \\
\hline Isotope & Year 1 & Year 30 & Associated Decay Chain \\
\hline $\mathrm{Xe}-132$ & 6.9792 & 6.4354 & ${ }^{132} I \stackrel{2 h}{\rightarrow}{ }^{132} \mathrm{Xe} \quad$ or $\quad{ }^{132} \mathrm{Cs} \stackrel{{ }^{6 d} \rightarrow{ }^{132} \mathrm{Xe}}{ }$ \\
\hline I-129 & 0.1482 & 0.1853 & ${ }^{129} \mathrm{Sb} \stackrel{24 h}{\longrightarrow}{ }^{129} \mathrm{Te} \stackrel{70 m}{\longrightarrow}{ }^{129} \boldsymbol{I}$ \\
\hline Te-125 & 0.0058 & 0.0525 & ${ }^{125} \mathrm{Xe} \stackrel{17 h}{\longrightarrow}{ }^{125} I \stackrel{59 d}{\longrightarrow}{ }^{125} \mathrm{Te}$ \\
\hline $\mathrm{I}-127$ & 0.0290 & 0.0501 & ${ }^{127} \mathrm{Cs} \stackrel{6 h^{127}}{\rightarrow} \mathrm{Xe} \stackrel{36 d}{\longrightarrow}{ }^{127} \boldsymbol{I}$ \\
\hline Te-126 & 0.0045 & 0.0042 & ${ }^{126} I \stackrel{13 d}{\longrightarrow}{ }^{126} T e$ \\
\hline Te-132 & 0.0949 & 0.0027 & ${ }^{132} \mathrm{Te} \stackrel{3 d^{132}}{\rightarrow} \stackrel{2 h}{\rightarrow}^{132} X e$ \\
\hline Te-129m & 0.0245 & 0.0011 & ${ }^{129 m} \mathrm{Te} \stackrel{70 m}{\longrightarrow}{ }^{129} I$ \\
\hline $\mathrm{Te}-127 \mathrm{~m}$ & 0.0202 & 0.0010 & ${ }^{127 m} \boldsymbol{T e} \stackrel{106 d}{\longrightarrow}{ }^{127} I$ \\
\hline $\mathrm{I}-132$ & 0.0028 & 0.0001 & ${ }^{132} I \stackrel{2.3 h}{\longrightarrow}{ }^{132} X e$ \\
\hline
\end{tabular}

$\underline{\text { SOFT - Fuel }}$

\begin{tabular}{|c|c|c|c|}
\hline \multirow[b]{2}{*}{ Isotope } & \multicolumn{2}{|c|}{ Concentration (mol\%) } & \multirow[b]{2}{*}{ Associated Decay Chain } \\
\hline & Year 1 & Year 30 & \\
\hline $\mathrm{I}-127$ & 0.0001 & 0.0020 & ${ }^{127} \mathrm{Cs} \stackrel{6 h^{h}}{\rightarrow}{ }^{127} \mathrm{Xe} \stackrel{36 d}{\longrightarrow}{ }^{127} \boldsymbol{I}$ \\
\hline Cs-137 & 0.0016 & 0.0017 & ${ }^{137} \mathrm{I} \stackrel{25 s}{\longrightarrow}{ }^{137} \mathrm{Xe} \stackrel{4 m^{\rightarrow}}{\rightarrow}{ }^{137} \mathrm{Cs} \stackrel{30 y}{\longrightarrow}{ }^{137} \mathrm{Ba}$ \\
\hline $\mathrm{I}-131$ & 0.0000 & 0.0010 & ${ }^{131} \mathrm{Te} \stackrel{25 m}{\longrightarrow}{ }^{131} I \stackrel{8 d}{\rightarrow}{ }^{131} \mathrm{Xe}$ \\
\hline Cs-135 & 0.0000 & 0.0001 & ${ }^{135} I \stackrel{7 h}{\rightarrow}{ }^{135} \mathrm{Xe} \stackrel{9 h}{\rightarrow}{ }^{135} \mathrm{Cs}$ \\
\hline
\end{tabular}

SOFT - Waste

\begin{tabular}{|c|c|c|c|}
\hline \multicolumn{3}{|c|}{ Concentration (mol\%) } & \multirow[b]{2}{*}{ Associated Decay Chain } \\
\hline Isotope & Year 1 & Year 30 & \\
\hline Cs-135 & 4.3377 & 4.2454 & ${ }^{135} \mathrm{I} \stackrel{7 h^{1}}{\rightarrow}{ }^{35} \mathrm{Xe} \stackrel{9 h}{\rightarrow}{ }^{135} \mathrm{Cs}$ \\
\hline Xe-134 & 3.9326 & 3.8148 & ${ }^{134} \mathrm{I} \stackrel{83 s}{\longrightarrow}{ }^{134} \mathrm{Xe} \quad$ or $\quad{ }^{134} \mathrm{Cs} \stackrel{2 y}{\rightarrow}{ }^{134} \mathrm{Xe}$ \\
\hline $\mathrm{Xe}-136$ & 3.5923 & 3.4996 & ${ }^{136} I \stackrel{83 s}{\longrightarrow}{ }^{136} \mathrm{Xe}$ \\
\hline Cs-133 & 3.2798 & 3.2600 & ${ }^{133} \mathrm{I} \stackrel{21 h}{\longrightarrow}{ }^{133} \mathrm{Xe} \stackrel{5 d}{\rightarrow}{ }^{133} \mathrm{Cs}$ \\
\hline Ba-138 & 2.2249 & 2.9975 & ${ }^{138} \mathrm{Xe} \stackrel{14 m}{\longrightarrow}{ }^{138} \mathrm{Cs} \stackrel{33 \mathrm{~m}}{\longrightarrow}{ }^{138} \mathrm{Ba}$ \\
\hline $\mathrm{Xe}-132$ & 2.6037 & 2.5838 & ${ }^{132} I \stackrel{2 h}{\rightarrow}{ }^{132} \mathrm{Xe} \quad$ or $\quad{ }^{132} \mathrm{Cs} \stackrel{6 d}{\rightarrow}{ }^{132} \mathrm{Xe}$ \\
\hline Cs-137 & 2.3467 & 2.3178 & ${ }^{137} \mathrm{I} \stackrel{25 s}{\longrightarrow}{ }^{137} \mathrm{Xe} \stackrel{4 m}{\rightarrow}{ }^{137} \mathrm{Cs} \stackrel{30 y^{137}}{\longrightarrow}{ }^{3}$ \\
\hline $\mathrm{Xe}-131$ & 1.6443 & 1.6560 & ${ }^{131} \stackrel{8 d}{\rightarrow}{ }^{131} \mathrm{Xe} \quad$ or $\quad{ }^{131} \mathrm{Cs} \quad \stackrel{10 d}{\longrightarrow}{ }^{131} \mathrm{Xe}$ \\
\hline Ba-137 & 0.0340 & 0.9055 & 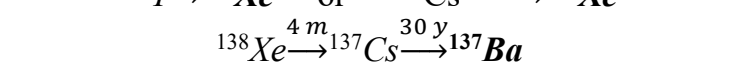 \\
\hline Sr- 88 & 0.5327 & 0.6954 & ${ }^{88} \mathrm{Kr} \stackrel{74 m}{\longrightarrow}{ }^{88} \mathrm{Rb} \stackrel{18 m}{\longrightarrow}{ }^{88} \mathrm{Sr}$ \\
\hline $\mathrm{Rb}-87$ & 0.4198 & 0.5503 & ${ }^{87} \mathrm{Br} \stackrel{56 s}{\longrightarrow}{ }^{87} \mathrm{Kr} \stackrel{{ }^{76 m}}{\longrightarrow}{ }^{87} \boldsymbol{R} \boldsymbol{b}$ \\
\hline $\mathrm{Kr}-86$ & 0.4638 & 0.4371 & ${ }^{86} \mathrm{Br} \stackrel{55 s}{\longrightarrow}{ }^{86} \mathrm{Kr} \quad$ or $\quad{ }^{87} \mathrm{Br} \stackrel{56 s}{\longrightarrow}{ }^{86} \mathrm{Kr} \quad$ or ${ }^{86} \mathrm{Rb} \stackrel{19 d}{\longrightarrow}{ }^{86} \mathrm{Kr}$ \\
\hline $\mathrm{Kr}-84$ & 0.2871 & 0.2734 & ${ }^{84} \mathrm{Br} \stackrel{32 m}{\longrightarrow}{ }^{84} \mathbf{K r} \quad$ or $\quad{ }^{84} R b \stackrel{33 d}{ }^{84} \mathbf{K r}$ \\
\hline $\mathrm{Rb}-85$ & 0.1687 & 0.2578 & ${ }^{85} \mathrm{Br} \stackrel{3 m^{85}}{\rightarrow} \mathrm{Kr} \stackrel{11 y^{85}}{\longrightarrow} \boldsymbol{B b}$ \\
\hline Kr-83 & 0.1657 & 0.1591 & ${ }^{83} \mathrm{Br} \stackrel{2 h}{\rightarrow}{ }^{83} \mathrm{Kr} \quad$ or $\quad{ }^{83} \mathrm{Rb} \stackrel{86 d}{\longrightarrow}{ }^{83} \mathbf{K r}$ \\
\hline $\mathrm{I}-129$ & 0.0895 & 0.1027 & ${ }^{129} \mathrm{Sb} \stackrel{24 h}{\longrightarrow}{ }^{129} \mathrm{Te} \stackrel{{ }^{70 m}{ }^{129} \mathrm{I}}{ }$ \\
\hline $\mathrm{Xe}-130$ & 0.0035 & 0.0521 & ${ }^{130} I \stackrel{12 h}{\rightarrow}{ }^{130} \mathrm{Xe} \quad$ or $\quad{ }^{130} \mathrm{Cs} \stackrel{29 m}{\longrightarrow}{ }^{130} \mathrm{Xe}$ \\
\hline
\end{tabular}




\begin{tabular}{|c|c|c|c|}
\hline $\mathrm{Kr}-85$ & 0.0703 & 0.0304 & ${ }^{85} \boldsymbol{K} \boldsymbol{r} \stackrel{11 y_{85}}{\longrightarrow} R b$ \\
\hline $\mathrm{I}-127$ & 0.0094 & 0.0154 & ${ }^{127} \mathrm{Cs} \stackrel{6 h^{127}}{\rightarrow} \mathrm{Xe} \stackrel{36 d}{\longrightarrow}{ }^{127} \boldsymbol{I}$ \\
\hline Te-125 & 0.0051 & 0.0398 & ${ }^{125} \mathrm{Xe} \stackrel{17 h^{125}}{\longrightarrow} \stackrel{59 d}{\longrightarrow}^{125} \mathrm{Te}$ \\
\hline $\mathrm{Xe}-128$ & 0.0003 & 0.0070 & ${ }^{128} I \stackrel{25 m}{\longrightarrow}{ }^{128} \mathrm{Xe}$ \\
\hline $\mathrm{Kr}-82$ & 0.0006 & 0.0046 & ${ }^{82} \mathrm{Br} \stackrel{35 h}{\longrightarrow}{ }^{82} \mathrm{Kr} \quad$ or $\quad{ }^{82} \mathrm{Rb} \stackrel{1 m}{\longrightarrow}{ }^{82} \mathbf{K r}$ \\
\hline Xe-133 & 0.0698 & 0.0023 & ${ }^{133} I \stackrel{21 h}{\longrightarrow}{ }^{133} \boldsymbol{X e} \stackrel{5 d}{\rightarrow}{ }^{133} \mathrm{Cs}$ \\
\hline Te-126 & 0.0014 & 0.0015 & ${ }^{126} I \stackrel{13 d}{\longrightarrow}{ }^{126} \mathrm{Te}$ \\
\hline Te-132 & 0.0331 & 0.0011 & ${ }^{132} \mathbf{T e} \stackrel{3 d}{\rightarrow}{ }^{132} I \stackrel{2 h}{\rightarrow}{ }^{132} \mathrm{Xe}$ \\
\hline Ba-140 & 0.0204 & 0.0007 & ${ }^{140} \mathrm{Xe} \stackrel{14 s}{\longrightarrow}{ }^{140} \mathrm{Cs} \stackrel{64 s}{\longrightarrow}{ }^{140} \mathrm{Ba}$ \\
\hline Te-129m & 0.0140 & 0.0005 & ${ }^{129 m} \mathrm{Te} \stackrel{70 m}{\longrightarrow}{ }^{129} I$ \\
\hline Te- $127 \mathrm{~m}$ & 0.0070 & 0.0003 & ${ }^{127 m} \mathrm{Te} \stackrel{106 d}{\longrightarrow}{ }^{127} I$ \\
\hline Xe-135 & 0.0058 & 0.0002 & ${ }^{135} I \stackrel{7 h}{\rightarrow}{ }^{135} \mathrm{Xe} \stackrel{9 h}{\rightarrow}{ }^{135} \mathrm{Cs}$ \\
\hline Te-124 & 0.0000 & 0.0001 & ${ }^{124} \stackrel{4 \underset{I}{\rightarrow}{ }^{124}}{ } \mathrm{Te}$ \\
\hline
\end{tabular}

\subsection{REACTOR PHYSICS}

The various isotopes that are of importance for neutronic purposes are identified in [13] for fission reactors and presented here in Tables 5 - 9 .

Table 5 Basic list of important nuclides for depletion calculations (15 isotopes).

\begin{tabular}{llll}
\hline${ }^{238} \mathrm{U}$ & ${ }^{234} \mathrm{U}$ & ${ }^{235} \mathrm{U}$ & ${ }^{236} \mathrm{U}$ \\
${ }^{240} \mathrm{Pu}$ & ${ }^{237} \mathrm{~Np}$ & ${ }^{238} \mathrm{Pu}$ & $\mathrm{Pu}$ \\
${ }^{242} \mathrm{Am}$ & ${ }^{241} \mathrm{Pu}$ & ${ }^{242} \mathrm{Pu}$ & ${ }^{241} \mathrm{Am}$ \\
& ${ }^{243} \mathrm{Am}$ & ${ }^{242} \mathrm{Cm}$ & ${ }^{243} \mathrm{Cm}$ \\
\hline
\end{tabular}


Table 6 First list of additional isotopes for depletion calculations. This list includes the 15 isotopes form Table 5 along with 49 additional isotopes (64 total).

\begin{tabular}{llll}
\hline${ }^{1} \mathrm{H}$ & ${ }^{10} \mathrm{~B}$ & ${ }^{11} \mathrm{~B}$ & \\
${ }^{14} \mathrm{~N}$ & ${ }^{16} \mathrm{O}$ & ${ }^{83} \mathrm{Kr}$ & ${ }^{93} \mathrm{Nb}$ \\
${ }^{94} \mathrm{Zr}$ & ${ }^{95} \mathrm{Mo}$ & ${ }^{99} \mathrm{Tc}$ & ${ }^{103} \mathrm{Rh}$ \\
${ }^{105} \mathrm{Rh}$ & ${ }^{106} \mathrm{Ru}$ & ${ }^{109} \mathrm{Ag}$ & ${ }^{126} \mathrm{Sn}$ \\
${ }^{135} \mathrm{I}$ & ${ }^{131} \mathrm{Xe}$ & ${ }^{135} \mathrm{Xe}$ & ${ }^{133} \mathrm{Cs}$ \\
${ }^{134} \mathrm{Cs}$ & ${ }^{135} \mathrm{Cs}$ & ${ }^{137} \mathrm{Cs}$ & $\mathrm{Pr}$ \\
${ }^{144} \mathrm{Ce}$ & ${ }^{143} \mathrm{Nd}$ & ${ }^{145} \mathrm{Nd}$ & ${ }^{146} \mathrm{Nd}$ \\
${ }^{147} \mathrm{Nd}$ & ${ }^{147} \mathrm{Pm}$ & ${ }^{148} \mathrm{Pm}$ & ${ }^{149} \mathrm{Pm}$ \\
${ }^{148} \mathrm{Nd}$ & ${ }^{147} \mathrm{Sm}$ & ${ }^{149} \mathrm{Sm}$ & ${ }^{153} \mathrm{Eu}$ \\
${ }^{151} \mathrm{Sm}$ & ${ }^{152} \mathrm{Sm}$ & ${ }^{151} \mathrm{Eu}$ & ${ }^{154} \mathrm{Gd}$ \\
${ }^{154} \mathrm{Eu}$ & ${ }^{155} \mathrm{Eu}$ & ${ }^{158} \mathrm{Gd}$ & \\
${ }^{155} \mathrm{Gd}$ & ${ }^{156} \mathrm{Gd}$ & & \\
${ }^{160} \mathrm{Gd}$ & ${ }^{244} \mathrm{Cm}$ & & \\
\hline
\end{tabular}

Table 7 Second list of nuclides for depletion calculations. This list includes the 64 isotopes from Table 6 and 31 additional isotopes (95 total) and serves as the current default for the Triton code.

\begin{tabular}{llll}
\hline${ }^{91} \mathrm{Zr}$ & ${ }^{93} \mathrm{Zr}$ & ${ }^{95} \mathrm{Zr}$ & \\
${ }^{95} \mathrm{Nb}$ & ${ }^{97} \mathrm{Mo}$ & ${ }^{96} \mathrm{Zr}$ & ${ }^{99} \mathrm{Mo}$ \\
${ }^{100} \mathrm{Mo}$ & ${ }^{101} \mathrm{Ru}$ & ${ }^{10} \mathrm{Ru}$ & ${ }^{103} \mathrm{Ru}$ \\
${ }^{104} \mathrm{Ru}$ & ${ }^{105} \mathrm{Pd}$ & ${ }^{107} \mathrm{Pd}$ & ${ }^{108} \mathrm{Pd}$ \\
${ }^{113} \mathrm{Cd}$ & ${ }^{115} \mathrm{In}$ & ${ }^{127} \mathrm{I}$ & ${ }^{129} \mathrm{I}$ \\
${ }^{133} \mathrm{Xe}$ & ${ }^{139} \mathrm{Ba}$ & ${ }^{141} \mathrm{Ce}$ \\
${ }^{142} \mathrm{Ce}$ & ${ }^{143} \mathrm{Ce}$ & ${ }^{141} \mathrm{Pr}$ & \\
${ }^{153} \mathrm{Sm}$ & ${ }^{156} \mathrm{Eu}$ & ${ }^{242 \mathrm{~m}} \mathrm{Am}$ & \\
\hline
\end{tabular}

Table 8 Third list of nuclides for depletion calculations. This list includes the 95 isotopes from Table 7 and 136 additional isotopes (231 total).

\begin{tabular}{llll}
\hline${ }^{72} \mathrm{Ge}$ & ${ }^{73} \mathrm{Ge}$ & ${ }^{74} \mathrm{Ge}$ & ${ }^{76} \mathrm{Ge}$ \\
${ }^{75} \mathrm{As}$ & ${ }^{79} \mathrm{Br}$ & ${ }^{77} \mathrm{Se}$ & ${ }^{82} \mathrm{Se}$ \\
${ }^{78} \mathrm{Se}$ & ${ }^{80} \mathrm{Se}$ & ${ }^{84} \mathrm{Br}$ & ${ }^{85} \mathrm{Kr}$ \\
${ }^{80} \mathrm{Kr}$ & ${ }^{82} \mathrm{Kr}$ & ${ }^{86} \mathrm{Rb}$ & ${ }^{87} \mathrm{Rb}$ \\
${ }^{86} \mathrm{Kr}$ & ${ }^{85} \mathrm{Rb}$ & ${ }^{87} \mathrm{Sr}$ & ${ }^{88} \mathrm{Sr}$ \\
${ }^{84} \mathrm{Sr}$ & ${ }^{86} \mathrm{Sr}$ & ${ }^{90} \mathrm{Y}$ \\
${ }^{89} \mathrm{Sr}$ & ${ }^{90} \mathrm{Sr}$ & ${ }^{92} \mathrm{Zr}$ & ${ }^{92} \mathrm{Mo}$ \\
${ }^{91} \mathrm{Y}$ & ${ }^{90} \mathrm{Zr}$ & ${ }^{94} \mathrm{Nb}$ & ${ }^{105} \mathrm{Ru}$ \\
${ }^{94} \mathrm{Mo}$ & ${ }^{96} \mathrm{Mo}$ & ${ }^{100} \mathrm{Ru}$ & ${ }^{110} \mathrm{Pd}$ \\
${ }^{98} \mathrm{Ru}$ & ${ }^{99} \mathrm{Ru}$ & ${ }^{106} \mathrm{Cd}$ & ${ }^{108} \mathrm{Cd}$ \\
${ }^{102} \mathrm{Pd}$ & ${ }^{104} \mathrm{Pd}$ & ${ }^{112} \mathrm{Cd}$ & ${ }^{114} \mathrm{Cd}$ \\
${ }^{107} \mathrm{Ag}$ & ${ }^{111} \mathrm{Ag}$ & ${ }^{140} \mathrm{Ce}$ & ${ }^{113} \mathrm{In}$ \\
${ }^{110} \mathrm{Cd}$ & ${ }^{111} \mathrm{Cd}$ & ${ }^{115} \mathrm{Sn}$ \\
\hline${ }^{115 m} \mathrm{Cd}$ & ${ }^{116} \mathrm{Cd}$ & & \\
${ }^{140} \mathrm{La}$ & ${ }^{112} \mathrm{Sn}$ & &
\end{tabular}




\begin{tabular}{|c|c|c|c|}
\hline$\overline{{ }^{116} \mathrm{Sn}}$ & ${ }^{117} \mathrm{Sn}$ & ${ }^{118} \mathrm{Sn}$ & ${ }^{119} \mathrm{Sn}$ \\
\hline${ }^{120} \mathrm{Sn}$ & ${ }^{122} \mathrm{Sn}$ & ${ }^{123} \mathrm{Sn}$ & ${ }^{124} \mathrm{Sn}$ \\
\hline${ }^{125} \mathrm{Sn}$ & ${ }^{121} \mathrm{Sb}$ & ${ }^{123} \mathrm{Sb}$ & ${ }^{124} \mathrm{Sb}$ \\
\hline${ }^{125} \mathrm{Sb}$ & ${ }^{126} \mathrm{Sb}$ & ${ }^{120} \mathrm{Te}$ & ${ }^{122} \mathrm{Te}$ \\
\hline${ }^{123} \mathrm{Te}$ & ${ }^{124} \mathrm{Te}$ & ${ }^{125} \mathrm{Te}$ & ${ }^{126} \mathrm{Te}$ \\
\hline${ }^{127 \mathrm{~m}} \mathrm{Te}$ & ${ }^{128} \mathrm{Te}$ & ${ }^{129 \mathrm{~m}} \mathrm{Te}$ & ${ }^{130} \mathrm{Te}$ \\
\hline${ }^{132} \mathrm{Te}$ & ${ }^{130} \mathrm{I}$ & ${ }^{131} \mathrm{I}$ & ${ }^{124} \mathrm{Xe}$ \\
\hline${ }^{126} \mathrm{Xe}$ & ${ }^{128} \mathrm{Xe}$ & ${ }^{129} \mathrm{Xe}$ & ${ }^{130} \mathrm{Xe}$ \\
\hline${ }^{132} \mathrm{Xe}$ & ${ }^{134} \mathrm{Xe}$ & ${ }^{136} \mathrm{Xe}$ & ${ }^{134} \mathrm{Ba}$ \\
\hline${ }^{135} \mathrm{Ba}$ & ${ }^{136} \mathrm{Ba}$ & ${ }^{137} \mathrm{Ba}$ & ${ }^{138} \mathrm{Ba}$ \\
\hline${ }^{136} \mathrm{Cs}$ & ${ }^{142} \mathrm{Pr}$ & ${ }^{142} \mathrm{Nd}$ & ${ }^{150} \mathrm{Nd}$ \\
\hline${ }^{151} \mathrm{Pm}$ & ${ }^{144} \mathrm{Sm}$ & ${ }^{148} \mathrm{Sm}$ & ${ }^{154} \mathrm{Sm}$ \\
\hline${ }^{152} \mathrm{Eu}$ & ${ }^{157} \mathrm{Eu}$ & ${ }^{232} \mathrm{U}$ & ${ }^{233} \mathrm{U}$ \\
\hline${ }^{159} \mathrm{~Tb}$ & ${ }^{160} \mathrm{~Tb}$ & ${ }^{160} \mathrm{Dy}$ & ${ }^{161}$ Dy \\
\hline${ }^{162} \mathrm{Dy}$ & ${ }^{163} \mathrm{Dy}$ & ${ }^{164} \mathrm{Dy}$ & ${ }^{165} \mathrm{Ho}$ \\
\hline${ }^{166} \mathrm{Er}$ & ${ }^{167} \mathrm{Er}$ & ${ }^{175} \mathrm{Lu}$ & ${ }^{176} \mathrm{Lu}$ \\
\hline${ }^{181} \mathrm{Ta}$ & ${ }^{182} \mathrm{~W}$ & ${ }^{183} \mathrm{~W}$ & ${ }^{184} \mathrm{~W}$ \\
\hline${ }^{186} \mathrm{~W}$ & ${ }^{185} \mathrm{Re}$ & ${ }^{187} \mathrm{Re}$ & ${ }^{197} \mathrm{Au}$ \\
\hline${ }^{231} \mathrm{~Pa}$ & ${ }^{233} \mathrm{~Pa}$ & ${ }^{230} \mathrm{Th}$ & ${ }^{232} \mathrm{Th}$ \\
\hline
\end{tabular}

Table 9 Final list of additional depletion nuclides. This list includes all isotopes from Tables $5-8$ and 158 additional nuclides (388 total).

\begin{tabular}{|c|c|c|c|}
\hline${ }^{2} \mathrm{H}$ & ${ }^{3} \mathrm{H}$ & ${ }^{3} \mathrm{He}$ & ${ }^{4} \mathrm{He}$ \\
\hline${ }^{6} \mathrm{Li}$ & ${ }^{7} \mathrm{Li}$ & ${ }^{7} \mathrm{Be}$ & ${ }^{9} \mathrm{Be}$ \\
\hline${ }^{15} \mathrm{~N}$ & ${ }^{17} \mathrm{O}$ & ${ }^{19} \mathrm{~F}$ & ${ }^{23} \mathrm{Na}$ \\
\hline${ }^{24} \mathrm{Mg}$ & ${ }^{25} \mathrm{Mg}$ & ${ }^{26} \mathrm{Mg}$ & ${ }^{27} \mathrm{Al}$ \\
\hline${ }^{28} \mathrm{Si}$ & ${ }^{29} \mathrm{Si}$ & ${ }^{30} \mathrm{Si}$ & ${ }^{31} \mathrm{P}$ \\
\hline${ }^{32} \mathrm{~S}$ & ${ }^{33} \mathrm{~S}$ & ${ }^{34} \mathrm{~S}$ & ${ }^{36} \mathrm{~S}$ \\
\hline${ }^{35} \mathrm{Cl}$ & ${ }^{37} \mathrm{Cl}$ & ${ }^{36} \mathrm{Ar}$ & ${ }^{38} \mathrm{Ar}$ \\
\hline${ }^{40} \mathrm{Ar}$ & ${ }^{39} \mathrm{~K}$ & ${ }^{40} \mathrm{~K}$ & ${ }^{41} \mathrm{~K}$ \\
\hline${ }^{40} \mathrm{Ca}$ & ${ }^{42} \mathrm{Ca}$ & ${ }^{43} \mathrm{Ca}$ & ${ }^{44} \mathrm{Ca}$ \\
\hline${ }^{46} \mathrm{Ca}$ & ${ }^{48} \mathrm{Ca}$ & ${ }^{45} \mathrm{Sc}$ & ${ }^{46} \mathrm{Ti}$ \\
\hline${ }^{47} \mathrm{Ti}$ & ${ }^{48} \mathrm{Ti}$ & ${ }^{49} \mathrm{Ti}$ & ${ }^{50} \mathrm{Ti}$ \\
\hline${ }^{50} \mathrm{Cr}$ & ${ }^{52} \mathrm{Cr}$ & ${ }^{53} \mathrm{Cr}$ & ${ }^{54} \mathrm{Cr}$ \\
\hline${ }^{55} \mathrm{Mn}$ & ${ }^{54} \mathrm{Fe}$ & ${ }^{56} \mathrm{Fe}$ & ${ }^{57} \mathrm{Fe}$ \\
\hline${ }^{58} \mathrm{Fe}$ & ${ }^{58} \mathrm{Co}$ & ${ }^{58} \mathrm{mCo}$ & ${ }^{59} \mathrm{Co}$ \\
\hline${ }^{58} \mathrm{Ni}$ & ${ }^{59} \mathrm{Ni}$ & ${ }^{60} \mathrm{Ni}$ & ${ }^{61} \mathrm{Ni}$ \\
\hline${ }^{62} \mathrm{Ni}$ & ${ }^{64} \mathrm{Ni}$ & ${ }^{63} \mathrm{Cu}$ & ${ }^{65} \mathrm{Cu}$ \\
\hline${ }^{70} \mathrm{Ge}$ & ${ }^{69} \mathrm{Ga}$ & ${ }^{71} \mathrm{Ga}$ & ${ }^{74} \mathrm{As}$ \\
\hline${ }^{74} \mathrm{Se}$ & ${ }^{79} \mathrm{Se}$ & ${ }^{78} \mathrm{Kr}$ & ${ }^{110 m} \mathrm{Ag}$ \\
\hline${ }^{113} \mathrm{Sn}$ & ${ }^{123} \mathrm{Xe}$ & ${ }^{130} \mathrm{Ba}$ & ${ }^{132} \mathrm{Ba}$ \\
\hline${ }^{133} \mathrm{Ba}$ & ${ }^{136} \mathrm{Ce}$ & ${ }^{138} \mathrm{Ce}$ & ${ }^{139} \mathrm{Ce}$ \\
\hline${ }^{138} \mathrm{La}$ & ${ }^{148 \mathrm{~m}} \mathrm{Pm}$ & ${ }^{153} \mathrm{Gd}$ & ${ }^{156}$ Dy \\
\hline${ }^{158} \mathrm{Dy}$ & ${ }^{166 m} \mathrm{Ho}$ & ${ }^{162} \mathrm{Er}$ & ${ }^{164} \mathrm{Er}$ \\
\hline${ }^{168} \mathrm{Er}$ & ${ }^{170} \mathrm{Er}$ & ${ }^{174} \mathrm{Hf}$ & ${ }^{176} \mathrm{Hf}$ \\
\hline${ }^{177} \mathrm{Hf}$ & ${ }^{178} \mathrm{Hf}$ & ${ }^{179} \mathrm{Hf}$ & ${ }^{180} \mathrm{Hf}$ \\
\hline${ }^{182} \mathrm{Ta}$ & ${ }^{191} \mathrm{Ir}$ & ${ }^{193} \mathrm{Ir}$ & ${ }^{196} \mathrm{Hg}$ \\
\hline
\end{tabular}




\begin{tabular}{llll}
\hline${ }^{198} \mathrm{Hg}$ & ${ }^{199} \mathrm{Hg}$ & ${ }^{200} \mathrm{Hg}$ & ${ }^{201} \mathrm{Hg}$ \\
${ }^{202} \mathrm{Hg}$ & ${ }^{204} \mathrm{Hg}$ & $204 \mathrm{~Pb}$ & ${ }^{206} \mathrm{~Pb}$ \\
${ }^{207} \mathrm{~Pb}$ & ${ }^{208} \mathrm{~Pb}$ & ${ }^{209} \mathrm{Bi}$ & ${ }^{226} \mathrm{Ac}$ \\
${ }^{222} \mathrm{Ra}$ & ${ }^{225} \mathrm{Ac}$ & ${ }^{228} \mathrm{Th}$ \\
${ }^{227} \mathrm{Ac}$ & ${ }^{226} \mathrm{Ra}$ & ${ }^{227} \mathrm{Th}$ & ${ }^{239} \mathrm{Na}$ \\
${ }^{229} \mathrm{Th}$ & ${ }^{233} \mathrm{Th}$ & ${ }^{234} \mathrm{Th}$ & ${ }^{241} \mathrm{U}$ \\
${ }^{235} \mathrm{~Np}$ & ${ }^{236} \mathrm{~Np}$ & ${ }^{240} \mathrm{U}$ & ${ }^{244} \mathrm{Pu}$ \\
${ }^{237} \mathrm{U}$ & ${ }^{239} \mathrm{U}$ & ${ }^{243} \mathrm{Pu}$ & ${ }^{244} \mathrm{Cm}$ \\
${ }^{236} \mathrm{Pu}$ & ${ }^{244} \mathrm{Am}$ & ${ }^{248} \mathrm{Cm}$ \\
${ }^{246} \mathrm{Pu}$ & ${ }^{246} \mathrm{Cm}$ & ${ }^{247} \mathrm{Cm}$ & $\mathrm{Bk}$ \\
${ }^{245} \mathrm{Cm}$ & ${ }^{250} \mathrm{Cm}$ & ${ }^{249} \mathrm{Bk}$ & ${ }^{252} \mathrm{Cf}$ \\
${ }^{249} \mathrm{Cm}$ & ${ }^{250} \mathrm{Cf}$ & ${ }^{251} \mathrm{Cf}$ & \\
${ }^{253} \mathrm{Cf}$ & & & \\
${ }^{255} \mathrm{Es}$ & & & \\
\hline
\end{tabular}

Table 8 includes the default selection for the radionuclides for LWR calculations using the TRITON code [13]. The proposed approach for mass accountancy modeling MSR behavior is to use those 95 isotopes not already identified in 2.1.1 as a starting point. Those presented in 9 form a comprehensive balance. These can be included for improving the accuracy of integrated multi-physics prediction for MSR modeling if necessary. Adding isotopes comes at computational expense; therefore, the cost vs benefit will be an important factor when considering the inclusion of a more comprehensive isotopic tracking inventory relevant to neutronics impacts. As previously stated in 2.1.1, isotopes with long half-lives (many actinides and certain fission products) may not undergo radioactive decay frequently enough to contribute significantly to changes in the fuel salt. These isotopes must still be included in the depletion calculations because of their interaction with the neutron flux but neglecting their radioactive decay may be a viable way to reduce the complexity and runtime of the mass accountancy modeling without sacrificing the needed accuracy.

\section{The Molten Salt Thermal Properties Database (MSTDB)}

Salt thermal properties are fundamental inputs needed for data-based mass accountancy modeling and simulation of MSRs. As pointed out in the Roadmap for thermal property measurement of Molten Salt Reactor systems [14], it is important to understand the variance in salt properties with composition. This is a challenge due to buildup of products generated from fission, transmutation, and decay but also from corrosion and additives. Previous efforts have used empirical relations to represent density ( $\rho$ ), viscosity $(v)$, thermal conductivity ( $\kappa)$, and specific heat or heat capacity $(\mathrm{Cp})$ as a function of composition and temperature predominately between pure salts. For mixtures relevant to MSRs with continuously this becomes a Sisyphean task as the compositional degrees of freedom are too large. Fortunately, the NEAMS program is developing the MSTDB. It is a practical path forward for data-based property and phase equilibria estimations of multicomponent salt mixtures derived from relations for the fundamental pseudo-binaries only.

It is well established with the materials thermodynamics community that multicomponent system behavior can be estimated by extrapolation from the lower order subsystems. The unaries and binaries are therefore fundamental. It has been demonstrated that two body interactions generally dominate [15] [16] [17]. The MSTDB is to be populated with pure and pseudo-binary ${ }^{3}$ salts with temperature and composition. To a first approximation, a formalism based on a Redlich-Kister expansion [18], or similar

\footnotetext{
${ }^{3}$ Since the end-members are halides, a two salt mixture is a pseudo-binary since the system is properly a ternary.
} 
expression [19] [20] [21], corrects for the interactions in binary systems that can be combined for predicting both thermodynamic behavior as well as $\rho, v, \kappa$, and $\mathrm{Cp}$ in higher order systems. In this way, populating the MSTDB with pure salt properties, and pseudo-binary mixture properties allows for databased extrapolation into multicomponent space, i.e. properties of base salt with fission products. An example of this for density is given in Figure 1. As systems are down-selected, specific compositions can be studied in more detail.

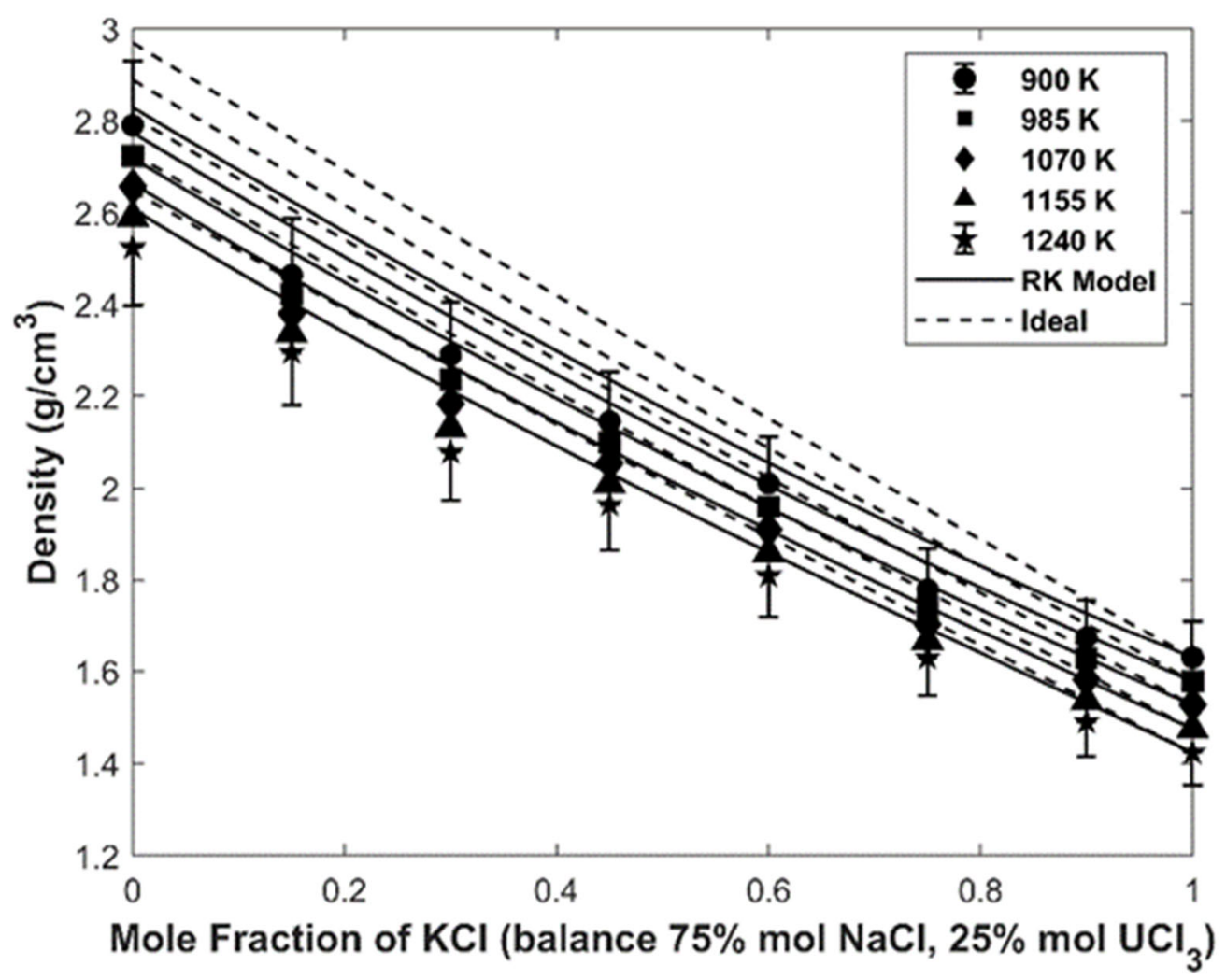

Figure 1 Calculated density for the pseudo-ternary NaCl-KCl-UCl3 system extrapolated from the pseudo-binaries.

The property data will be generated experimentally and computationally, e.g. via ab-initio and classical Molecular Dynamics (MD). This raw data are used as inputs for development of the MSTDB by optimizing the adjustable parameters to either a Gibbs energy model or an empirical one for representing $\rho, v, \kappa$, and $\mathrm{Cp}$ in temperature and composition (T-C) space. The MSTDB is subdivided into a thermochemical (MSTDB-TC) and a thermophysical database (MSTDB-TP). This is done because of the fundamental difference in how the two are used as inputs. A Gibbs Energy Minimizer (GEM) is needed to calculate thermodynamic equilibrium for predicting phase relations and other values like vapor pressure ( $\left.\mathrm{p}^{*}\right), \mathrm{Cp}^{4}$ and chemical potentials using the MSTDB. The NEAMS program is developing the Yellowjacket GEM to be discussed in more detail later.

\footnotetext{
${ }^{4}$ The specific heat or heat capacity is sometimes considered a thermophysical property. It is a thermodynamic property and can be calculated using Maxwell relations from the Gibbs energy.
} 


\subsection{Thermochemical properties}

The Calculation of Phase Diagram (CALPHAD) method [22] is the accepted approach for thermodynamic modeling and database development within the materials community. The MSTDB-TC is developed from existing literature, newly generated experimental data primarily from the MSR campaign, and computational derived properties using classical or ab-initio Molecular Dynamics. An accompanying data package documenting the source of every model and/or the raw data used for its continuing development is included [23]. Currently, it accommodates at least 21 elements and models for 63 pseudo-binary (47 fluoride, 16 chloride) and 29 pseudo-ternary ( 28 fluoride, 1 chloride) molten salt solutions along with 26 solid solutions and 89 stoichiometric compounds. In addition there are 5 higher order chloride systems assessed. With CALPHAD, the database can be built upon, or extended, to include more elements as more systems are studied. The aim is to include all the impactful elements that will exist during operation of an MSR including system components for alloys that contact the salt to model the thermochemical behavior with burnup including additives and corrosion products. The MSTDB-TC contains the information necessary to compute inputs, e.g. phase equilibria and chemical potentials, for multi-physics codes to predict mass accountancy including microstructure and mass transport in structural materials.

\subsection{Thermophysical properties}

The MSTDB-TP is the thermophysical analogue to MSTDB-TC. It is a collection of empirical models for representing $\rho, v, \kappa$, and $\mathrm{Cp}$ of molten salts as a function of temperature and composition. These are required inputs for thermal hydraulics and mass transport models. Unlike the MSTDB-TC, it does not require a GEM. Models are additive based on a mechanical mixture of the pure salt compound constituents with binary interactions only. In some cases, when available, ternary or higher order interaction parameters may be included. Work continues an API for standalone material properties predictions and the Saline code for coupling to Multiphysics codes to be discussed in more detail in Section 3.5. Currently there are 62 entries. Of them, 27 are pure compounds (14 fluorides and 13 chlorides), 8 pseudo-binary systems ( 1 chloride and 7 fluorides), 10 pseudo-ternaries (all of them fluorides) and 5 pseudo-quaternaries (all of them fluorides).

\subsection{Computationally derived thermal properties}

A plan for fully developing the MSTDB based on system of interest based on input from developers and the NRC is presented in [14] An important component of the plan is the use of computational techniques like ab-initio and classical MD for property predictions. The NEAMS program is developing the tools necessary for predict density $(\rho)$, viscosity $(v)$, thermal conductivity $(\kappa)$, and specific heat $(\mathrm{Cp})$. Recently, both chloride and fluoride system properties have been calculated and benchmarked to existing data. Results for $\mathrm{NaCl}, \mathrm{UCl}_{3} / \mathrm{UCl}_{4}$, and $\mathrm{NaCl}-\mathrm{UCl}_{3}$ are shown in Figures 2-4. The approach has been demonstrated to effectively represent properties like for FLiBe (eutectic LiF-BeF 2 ), FLiNaK (46.5-11.5$42 \mathrm{~mol} \% \mathrm{LiF}-\mathrm{NaF}-\mathrm{KF}$ eutectic), and $\mathrm{NaCl}-\mathrm{UCl}_{3}-\mathrm{UCl}_{4}$ compositions.

Using MD presents an opportunity to quickly predict pure salt and pseudo-binary molten salt relations with temperature and composition (T-C). These data can then be validated with targeted measurements and/or over T-C regions of specific interest to stakeholders. Properties are calculated for pure compounds and pseudo-binary mixtures over an extensive, continuous T-C space; validated experimentally; then implemented into MSTDB-TP. Once sufficient fundamental subsystem data exists, multicomponent assemblages are predicted. The MSTDB-TP empirical models can be further refined through subsequent targeted measurements. 


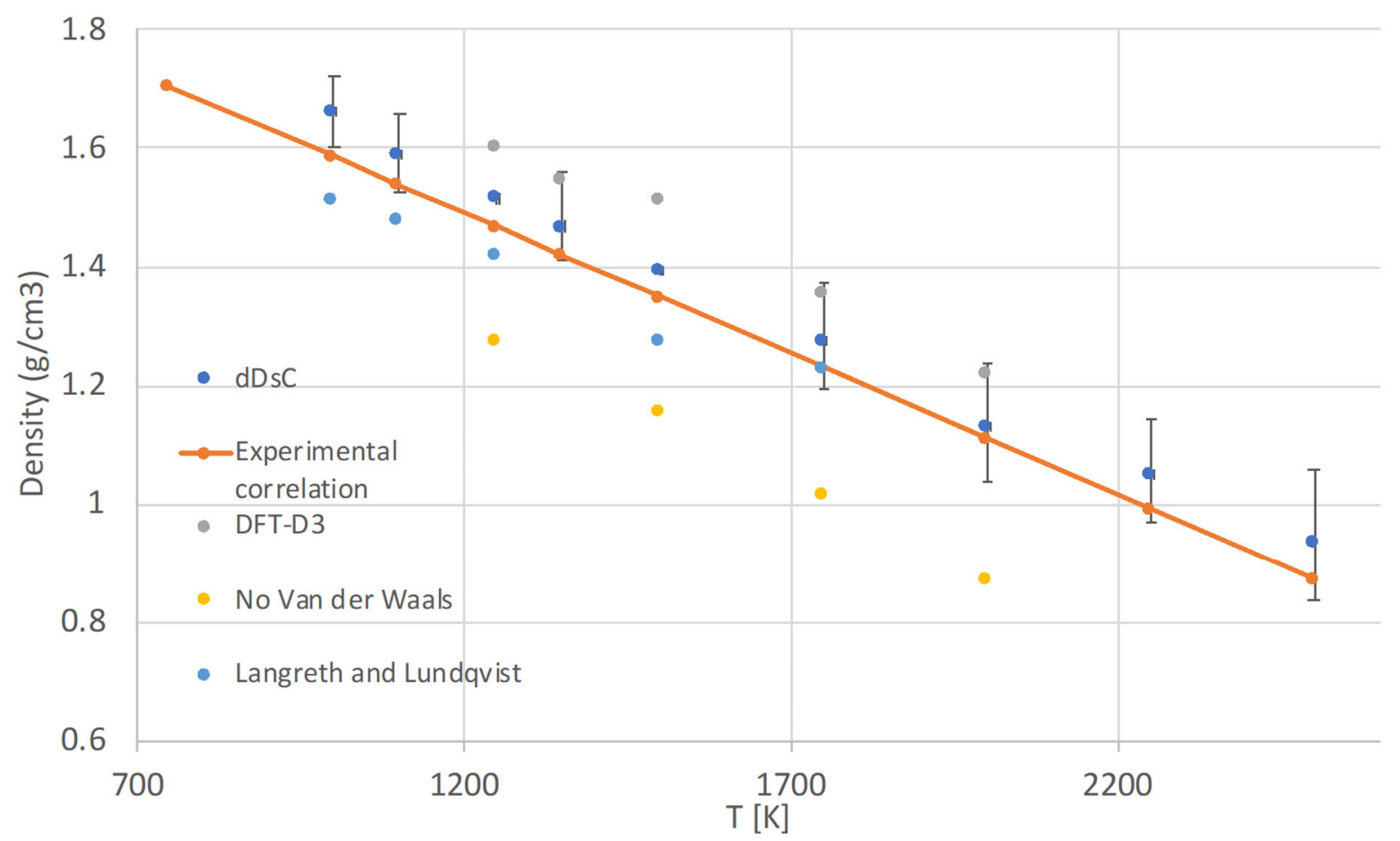

Figure $2 \mathrm{NaCl}$ denity with temperature computed using AIMD compared to experimental measurments. 


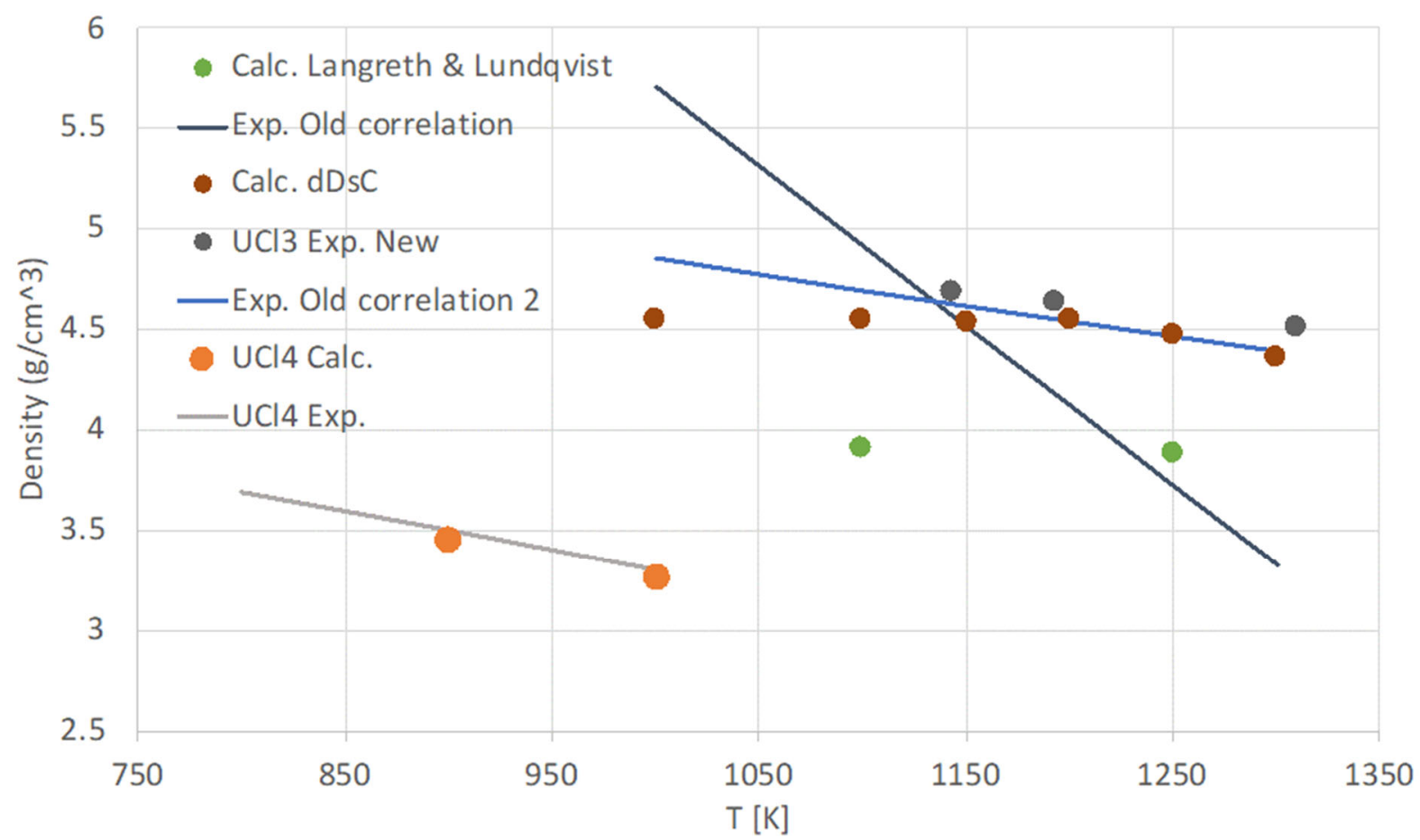

Figure 3 The density of $\mathrm{UCl}_{3}$ and $\mathrm{UCl}_{4}$ with temperature computed using AIMD compared to experimental measurments.

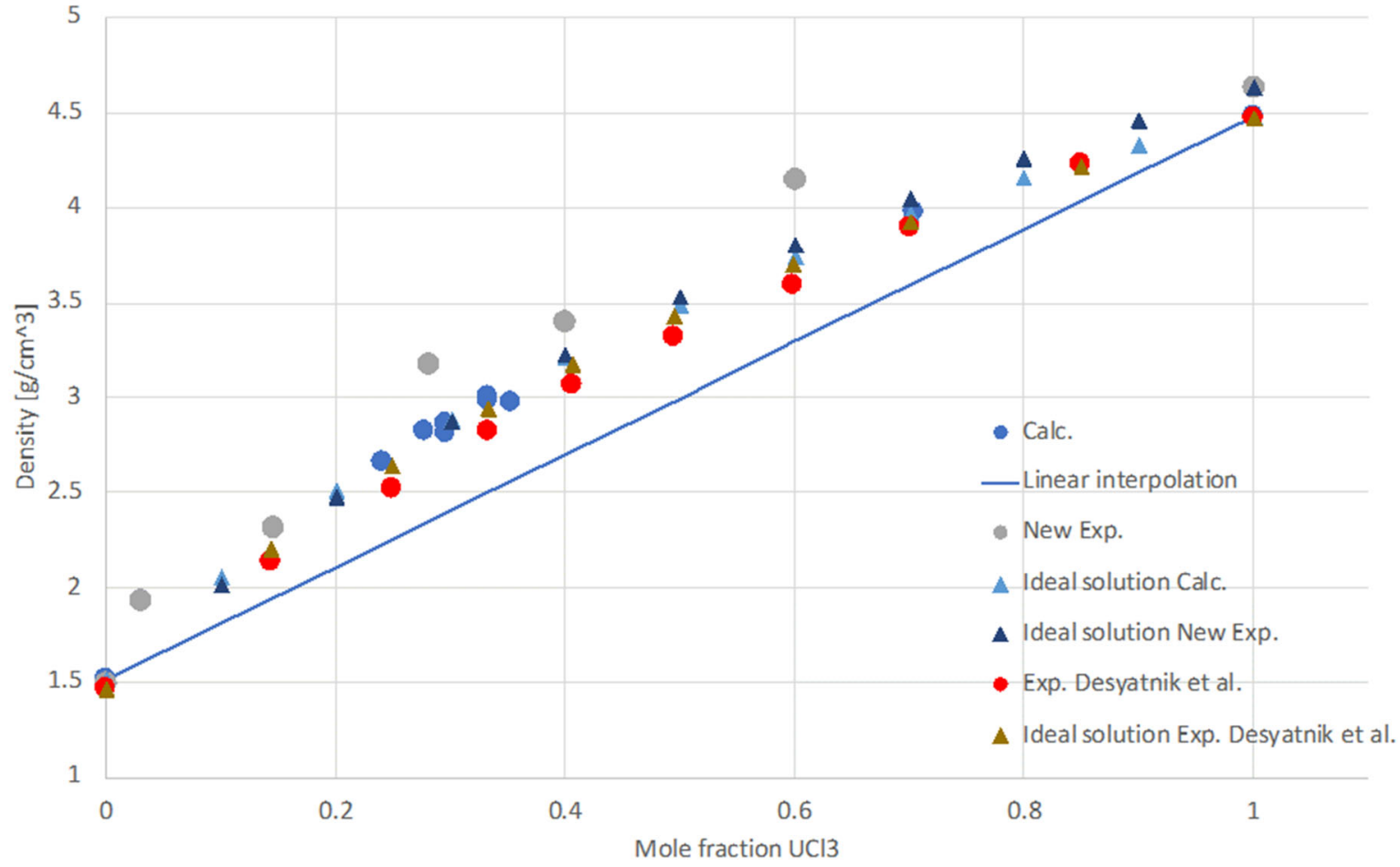

Figure 4 A mixture of $\mathrm{NaCl}-\mathrm{UCl}_{3}$ at $X X X^{\circ} \mathrm{C}$ computed using AIMD and compared to experimental data. 


\subsection{Gibbs Energy Minimization}

GEM tools are used to compute thermodynamic equilibria, which can predict which phases are stable at equilibrium, their speciation, and other thermodynamic properties. Applications range from the construction of phase diagrams to predicting complex chemical behavior like corium under severe accident conditions. Historically, most GEM software have been commercial tools, which limit their ability to couple to other software in a multi-physics framework. Computational performance of commercial thermodynamic software has been an issue. Also, most of these tools have not been developed for nuclear materials, which pose computational challenges mainly related to the system size.

Progress has been made to develop a modern, high-performance, quality assured software tool called Yellowjacket-GEM for coupling to multi-physics, multi-scale codes in MOOSE that could also be used with other applications outside of the MOOSE framework [24]. It is intended to support modelling and simulation relevant to nuclear fuel systems (i.e., molten salts, oxides, nitrides, alloys, etc.). For the current project, Yellojacket-GEM is needed to utilize the MSTDB to simulate molten salt behavior. The current status of this software development is that it can handle several solution model types to fully support the MSTDB and a prototypic version of the solver has been developed, but work is needed to extend its capabilities and continue testing to ensure convergence is not an issue. Furthermore, some coupling with MARMOT will be performed to inform meso-scale simulations of molten salt corrosion. This work is expected to be completed in FY21/22.

Longer term applications of Yellowjacket-GEM include coupling with other NEAMS tools, which may include Mole for MSR applications, BISON for LWR, TRISO or metallic fuels, or as a stand-alone tool. Example applications that one could expect is coupling to engineering tools, for example CFD and systems codes like SAM, to simulate phase transformations with mass transport to provide radioisotope distribution predictions for source term analyses and initial conditions for severe accident scenarios as well as to support safeguard analyses. Future developments may also include the development of a GUI to be more user-friendly, which would enable users to better work with and continue development of the MSTDB, generate phase diagrams, perform point calculations, etc. One application includes model development to support MSTDB development as the OptiSage tool that has been historically used is often reported to be problematic.

\subsection{Silane Application Programming Interface}

The Saline API seeks to provide a useful quality assured interface for obtaining density, viscosity, thermal conductivity, and/or specific heat capacity of various salt compositions across a range of temperatures. To facilitate ease of use Saline is compiled with validated data sources from open literature as described in Section 3.2. However, it also allows for providing standalone data assuming it conforms to input specifications.

Python bindings of the API have been developed to facilitate testing and development work and Fortran bindings have been developed to support potential HPC client applications. Additional user interfaces, up to and including GUIs, could be developed as required. 


\section{Modeling and simulation of mass accountancy}

Modeling of mass accountancy in MSRs requires a multi-physics approach. Thermodynamic values, e.g. chemical potentials, are the driving forces for phase transformations and mass transport. Thermophysical properties are fundamental to thermal hydraulic modeling and simulation. Neutronics calculations provide the isotopic inventory for identifying the species essential to understanding the chemical state determined by the thermodynamics and temporal and spatial location of each isotope that is influenced by the kinetics of mas transfer and phase transformation rates. Figure 5 shows how these phenomena are inextricably linked to the broader reactor physics and thermal hydraulic behavior of an MSR core and loop.

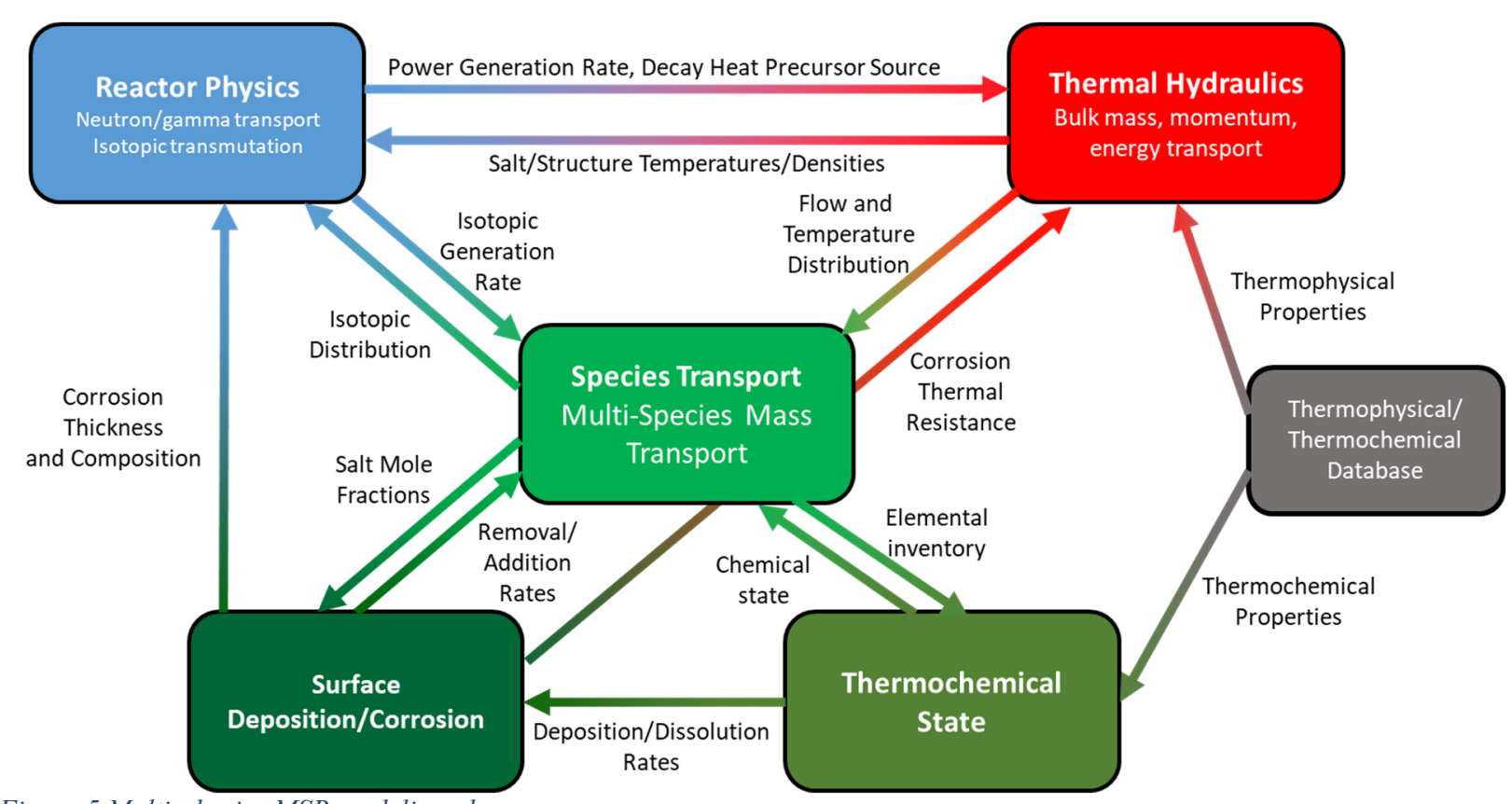

Figure 5 Multi-physics MSR modeling phenomena

To summarize, reactor physics gives the isotopic inventory, where the heat is generated, neutron transport behavior. Thermal hydraulics predicts how the heat is distributed and flow velocity fields. Reaction kinetics and phase transformations determine where precipitates deposit, vaporization rates, and erosion behavior. Fundamental inputs for broad based MSR isotopic tracking is phase equilibria and chemical potentials provided by thermodynamics along with the compositional dependent densities, viscosities, thermal conductivity and specific heat of the salt.

To address the challenge of integrating the multi-physics nature of mass accountancy for MSR, the following suite of codes proposed in Figure 6. Most of the codes are developed using MOOSE, that is a framework providing an array of tools for solving partial differential equations (PDEs) utilizing the finite element method (FEM) for a variety of different physics applications. This includes FEM meshing utilities, time-integration and physics kernels, mesh adaptivity, automatic differentiation, and flexible user inputs to model specific physics. MOOSE is designed to allow development of new physics applications focusing only on the specific physics kernels for their applications without worrying about many details that normally go into the development of a physics code. Additionally, it is also designed for multiphysics coupling, as the name implies, making it straightforward to model physics which have multiple variables that depend on each other. 
Finally, the overall NEAMS plan for modeling and simulation of MSRs spans length scales since abinitio Molecular Dynamics (AIMD) is used to aid develop the MSTDB by generating thermophysical properties and thermodynamic values.

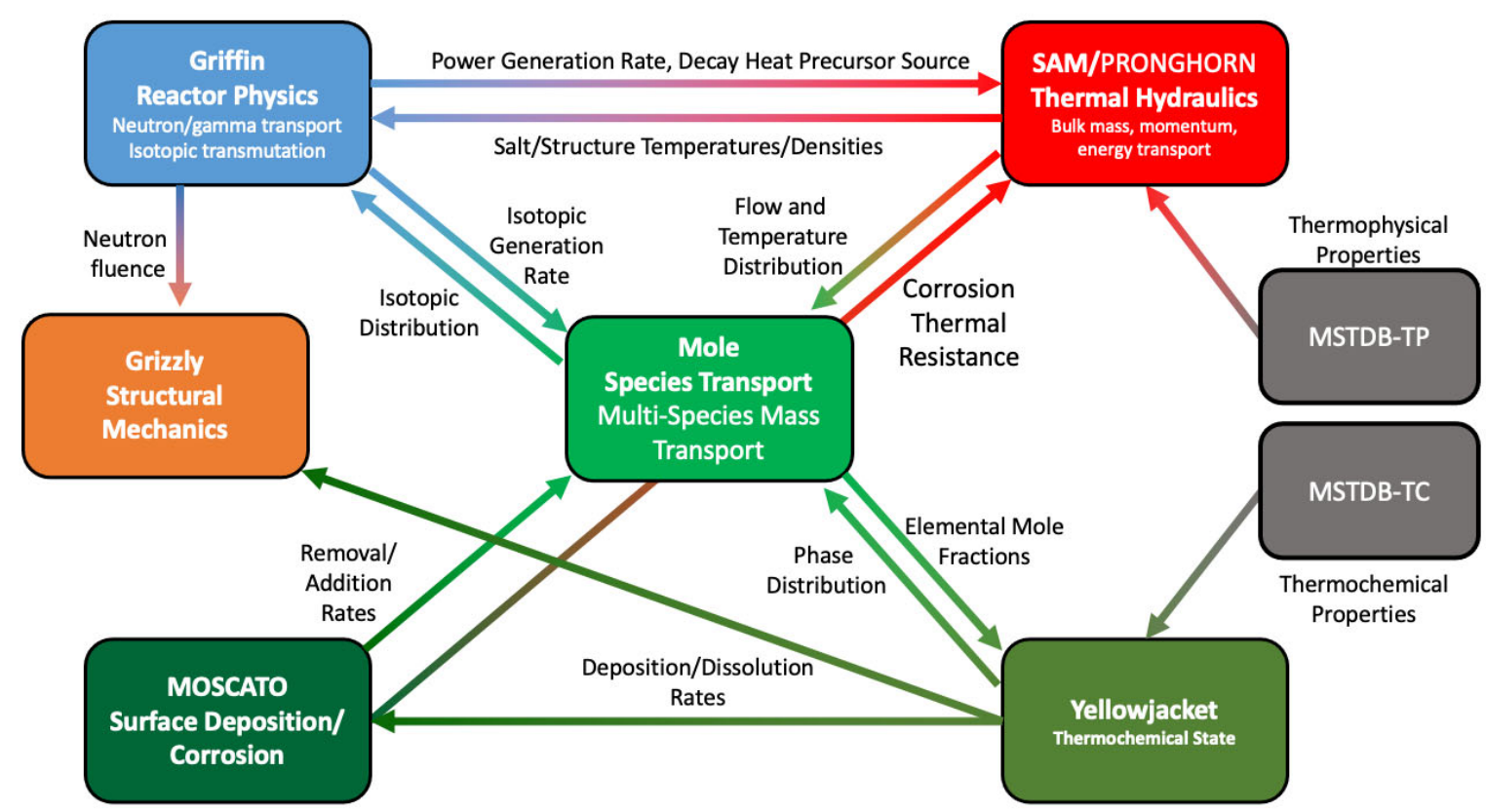

Figure 6 NEAMS multiphysics code suite for MSR mass accountancy.

\subsection{Neutronics}

Neutron interactions play a key part in fission product generation terms for mass accountancy. Coupling existing neutronics, cross section and depletion codes will be needed to accurately model the fission product inventory as a function of time. NEAMS tools such as Griffin can be coupled with species transport codes, such as Mole, to provided averaged neutron flux and cross-section data which are used in modeling the fission product reaction rates in the molten salt reactor core.

\subsection{CFD}

Argonne's MOSCATO code is a high-fidelity multiphysics solver capable of coupled fluid flow, heat transfer, mass transfer, electrochemistry, and alloy simulations for large-scale MSR equipment. The existing solver is based on the msrChemistryFoam solver that was developed to perform simulations of nuclear fuel reprocessing equipment [25].

The solver will be used to calculate local and integrated mass transfer coefficients for complex geometries, including the heat exchanger, pump bowl, etc. During the time of the MSRE, these types of simulations were untenable, and rough estimates of the mass transfer behavior were instead used to facilitate mass accountancy studies [26]. Since then, vast increases in capabilities for computational fluid dynamics (CFD) and multiphysics modeling have made simulations of challenging molten salt equipment achievable. For example, in FY20 the solver was used to perform engineering-scale simulations of chromium depletion in demonstration thermal convection loops, as shown in Figure 7. These plots show 
the rates of chromium transfer (depletion in hot regions and deposition in cold regions) resulting from the thermogalvanic phenomena.

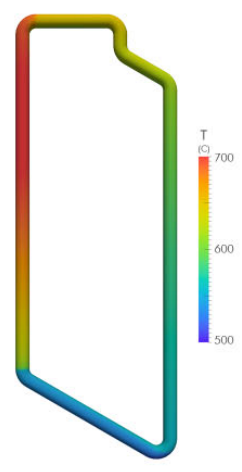

temperature

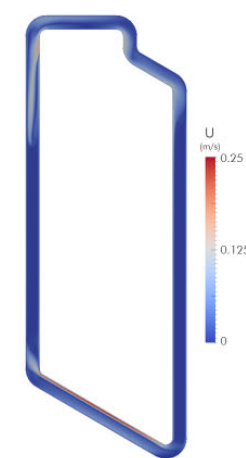

velocity magnitude (midplane)
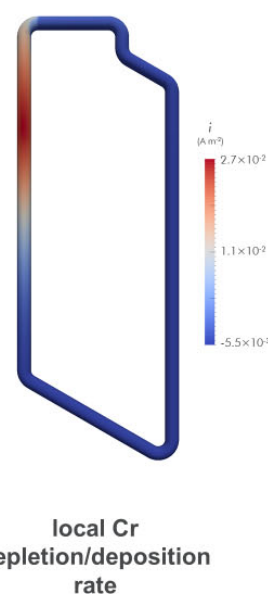

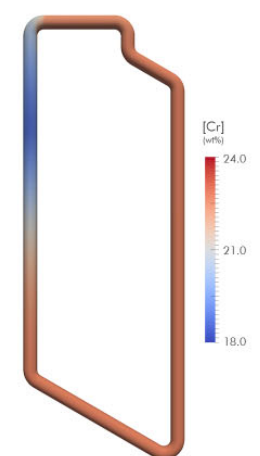

$\mathrm{Cr}^{0}$ concentration in alloy at salt interface

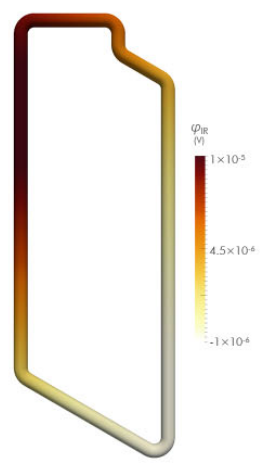

Ohmic overpotential from current flow in pipe walls

Figure 7 Simulated results for a thermal convection loop demo case (a) Temperature field (b) Velocity field (on the midplane) (c) Local corrosion rate (d) Concentration of $\mathrm{Cr}^{0}$ in alloy at salt interface (e) Ohmic overpotential associated with electron transfer in structural metal walls.

These high-fidelity mass transfer coefficients will in turn be used by as part of the YellowJacket corrosion suite to calculate sources and sinks for various species including noble metals and corrosion products. The current solver is written in OpenFOAM, an open-source framework for finite volume calculations. In order to permit integration with the MOOSE framework and to allow simulations of larger-scale MSR systems, MOSCATO is being transitioned to nekRS, a highly parallelizable spectral-element code that makes use of the latest generation of GPU-accelerated capabilities.

\subsection{Kinetics of reactions and mass transport}

Mole is a new code for solving species transport problems for mass accountancy modeling in a fluid circuit on the engineering scale. This includes a wide variety of phenomena including leaching from a solid interface, deposition of species onto surfaces, gas bubble and particulate formation, vapor-liquid transport, and others. The code is built to be flexible, allowing for a wide range of different species to be simulated over a wide variety of time scales. Mole can be utilized as submodule that will fit into Pronghorn and SAM or similar CFD code.

\subsubsection{Leaching and deposition}

In FY20 the capability to model leaching and deposition was demonstrated and benchmarked to experimental corrosion studies for $\mathrm{Cr}$ in $\mathrm{NaCl}-\mathrm{KCl}-\mathrm{MgCl}_{2}$ for $\mathrm{Ni}-\mathrm{Cr}$ alloys.

Suspended particles are not directly modeled, only the rate of deposition based on the models from the analytical approach from Kedl [26]. 


\subsubsection{Erosion}

Erosion can be life limiting especially if the salt contains suspended particulates. Do the noble fission products agglomerate into microparticles prior to depositing? As salt is expensive, there is an economic incentive to flow as fast as possible to minimize the fuel salt volume. We have no idea when erosion becomes a problem with real salt, so can't provide any guidance maximum safe flow velocity.

\subsubsection{Gas phase behavior}

Liquid-gas transport was found to be an important mechanism for understanding xenon poisoning in the MSRE [27]. In FY21 the capability to model the transport of generic chemical/radionuclide species from a liquid to a gas phase. These physics models aim to model the process of gas sparging via an injected gas and secondary removal system. Mole will provide the physics kernels which can be used standalone or coupled with existing MOOSE based applications which provide more detailed auxiliary physics.

The reactor core, moderator and reflectors of many molten salt reactor designs call for use of graphite that can be permeated by molten impacting microstructural properties, radiation behavior as well as radioisotope distribution. Material tests from the Molten Salt Reactor Experiment (MSRE) revealed a highly radioactive layer on or in the graphite with tritium $(\mathrm{T})$ and other fission products much deeper that likely penetrated the graphite as noble gases [28] [29]. It will be important to model mass transport of vapor species in order to develop predictive capabilities of radioisotope distribution in permeable media like graphite.

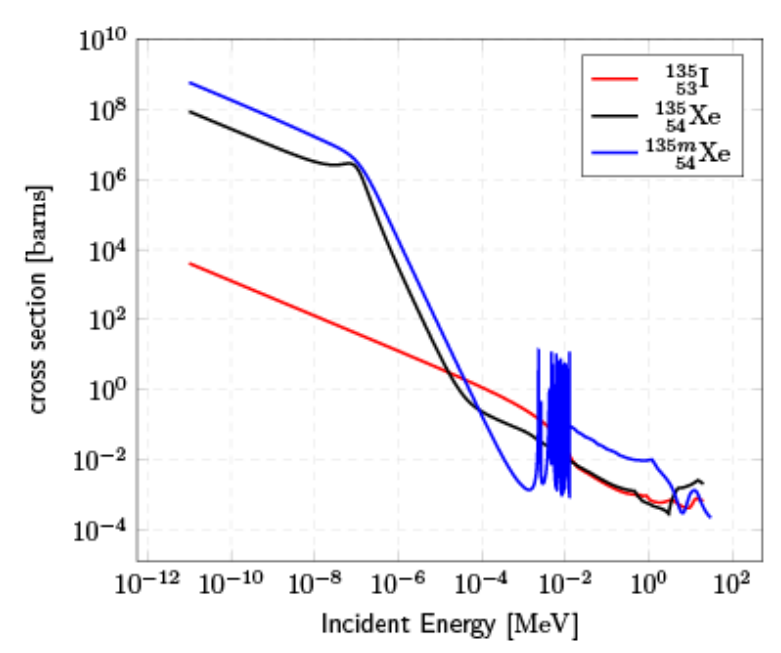

Figure 7 The microscopic cross section of absorption.

The microscopic cross-section of absorption of xenon is shown above the figure. The cross-section of ${ }_{54}^{135 m} \mathrm{Xe}$ is presented with ${ }_{53}^{135} \mathrm{I}$ and ${ }_{54}^{135} \mathrm{Xe}$. The concentration of ${ }_{54}^{135 m} \mathrm{Xe}$ can contribute to ${ }_{54}^{135} \mathrm{Xe}$ despite its relatively short half-life, ${ }_{54}^{135 m} \mathrm{Xe} \rightarrow{ }_{54}^{135} \mathrm{Xe}$. There are influencing factors by the fission fraction and the neutron activations of the Molten Salt Reactor. The decay chain of ${ }_{54}^{135 m} \mathrm{Xe}$ was not usually considered. 


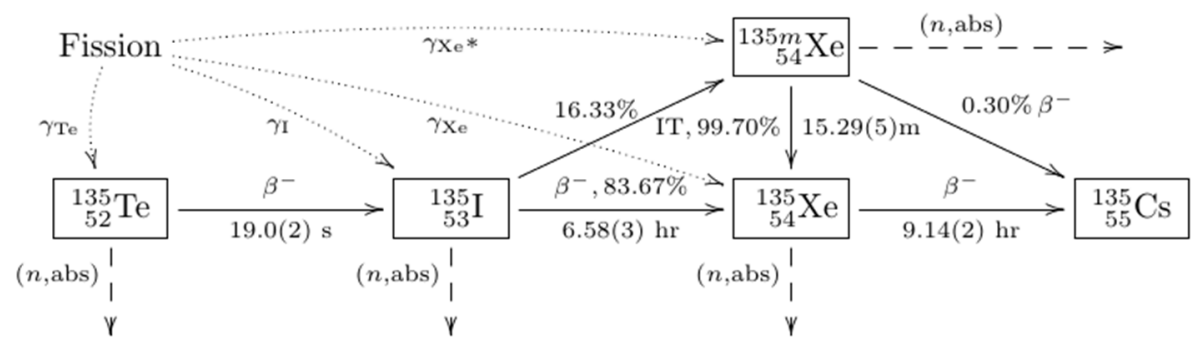

Figure 8 Decay chain of ${ }_{54}^{135 m}$ Xe and ${ }_{54}^{135}$ Xe from ENSDF data.

The figure is shown with meta-stable ${ }_{54}^{135 m} \mathrm{Xe}$ from ENSDF data. Although the sum of the branching ratio, $\beta$-of ${ }_{53}^{135} \mathrm{I}$, referred $100.3 \%$ in the Evaluated Nuclear Structure Data File (ENSDF) due to a rounding error and the decay of ${ }_{53}^{135} \mathrm{I}$ is related to the pandemonium effect for the isomeric state ${ }_{54}^{135 m} \mathrm{Xe}$, the sum of intensity (\%) from ${ }_{53}^{135} \mathrm{I}$ to ${ }_{54}^{135} \mathrm{Xe}$ is $83.67 \%$ in ENSDF. The branching ratio of the isomeric state can significantly affect the calculation for the concentration of ${ }_{54}^{135} \mathrm{Xe}$. The $16.33 \%$ branching ratio of ${ }_{53}^{135} \mathrm{I}$ can affect the bubble effect. The concentration of ${ }_{54}^{135} \mathrm{Xe}$ can be investigated as the effect of the decay chain with or without ${ }_{54}^{135 m} \mathrm{Xe}$.

The bubbling effects of noble gas, such as liquid-gas transport of xenon and krypton, cause the rate of mass transport of other species to change in the salt cycle of MSRE. The concentration of noble gas plays an important role in the overall system. The mass transfer depositions of noble metals affect all salt cycling

\subsection{SAM and thermal hydraulics}

The System Analysis Module (SAM) is a modern system analysis tool under development at Argonne National Laboratory for advanced non-LWR safety analysis. It aims to provide fast-running, improvedfidelity, whole-plant transient analyses capabilities, which are essential for fast turnaround design scoping and engineering analyses of advanced reactor concepts. SAM takes advantage of advances in physical modeling, numerical methods, and software engineering, to enhance its user experience and usability. While SAM is being developed as a system-level modeling and simulation tool, advanced modeling capabilities include a reduced-order three-dimensional flow module, fluid solidification, pseudo 3-D conjugate heat transfer modeling in reactor core, flexible multi-scale heat transfer between fluid and structures, and various reactor specific modeling features.

The ability to track various species (such as delayed-neutron precursors and decay heat precursors) in the primary reactor loop system is key for MSR transient simulation. For this purpose, the mass transport modeling capability has already been implemented in SAM based on the general scalar transport model in the fluids, in which the advection, diffusion, decay, and source terms are considered. Additionally, the initial tritium transport modeling capability has been implemented in SAM in collaboration with Kairos Power to support FHR source term evaluation. The detailed material models in Mole such as chemical reaction, precipitation, corrosion, or mass transfer kinetics at the fluid-solid interfaces, can be used in SAM as source/sink terms or closure models for system level mass transport modeling. It is important to integrate computationally efficient yet accurate lumped parameter material models into system-level analysis code for MSR mass accountancy modeling. Leveraging its capabilities in describing and modeling reactor systems and components, SAM will be the ideal platform to solve the system-level mass accountancy in MSRs. 


\subsubsection{Gas transport}

The species transport capability in SAM was expanded to include tracking of a gas phase, which may be encountered in MSR designs and play a role in the system behavior. In the Molten Salt Reactor Experiment (MSRE) performed at Oak Ridge National Laboratory, for example, helium gas was entrained in the system, which impacted fission gas collection and removal. In addition to tracking the gas species, which can be performed by the general species tracking model in SAM, models were also needed for prediction of gas volume fraction and interfacial area, which are important for the calculation of the rate of species transport between liquid and gas phases to be performed by the Mole code. In addition to these closure models, a simple drift flux model was also added to capture the potential for the gas phase to move at a different velocity than the bulk liquid. This drift velocity will be used to transport the species when that species is a gas, which will impact the gas species distribution in the system.

\subsection{Pronghorn}

Pronghorn is a multidimensional, coarse-mesh, thermal-hydraulics (TH) code for advanced reactors. It balances intermediate fidelity between detailed computational fluid dynamics (CFD) analysis and lumped system models. It can be useful when multidimensional fluid dynamics are required, for example modeling an MSR core. It can be coupled to SAM, Mole, and Yellowjacket to model the balance of the loop outside of the core region.

\subsection{Structural materials}

Leaching from structural materials by the molten salt degrades the mechanical behavior of the structural material and acts as a source of impurities for the molten salt. Predicting this behavior is a multi-scale, multi-physics problem spanning reactor physics, chemistry, and structural mechanics and from meso- to engineering scale. The Grizzly structural mechanics code will need materials models that predict this leaching. However, the leaching rate is directly impacted by the microstructure of the material and must be investigated at the mesoscale. As discussed, Yellowjacket-PF is a mesoscale phase field [30] [31] [32] code for solid-state mass transport simulating diffusion processes, microstructural evolution, and pore formation using thermodynamic inputs, specifically chemical potentials and phase equilibria. It predicts the impact of alloy composition and microstructure on the transport of elements from the structural material into the molten salt. The aim is for Yellowjacket-PF is to utilize the MOOSE framework to couple to the GEM and Mole, SAM, and Griffin [2] [3]. Yellowjacket-PF provides kinetic information for engineering-scale modeling with Grizzly that, in turn, provides sink terms that arise from the dynamic nature of a flowing loop to Yellowjacket-PF. Yellowjacket-PF is the coupling point between the chemistry and continuum mechanics codes by providing compositional profiles and microstructure, both of which dominate structural material behavior.

\subsubsection{Structural material leaching and deposition}

There will be consequences for material deposition on structural alloys. For example, one reason for keeping the salt reducing is to maintain Te dissolved in the salt. Tellurium is known to cause surface cracking of Hastelloy N [33] [34] [35]. It is also important to have the capability to model deposition of noble metals and other fission products on the alloy surfaces as there may be other adverse or advantageous reactions that happen over time/temperature due to these processes. 


\subsubsection{Radiation induced embrittlement}

Radiation induced embrittlement can be the life limiting phenomena and should be modeled for performance predictions. A known example is He embrittlement, which is especially problematic for nickel-based alloys that are irradiated at high temperature, that is above $500^{\circ} \mathrm{C}$. Helium can be formed with thermal neutrons. The He atoms migrate to the grain boundary resulting in embrittlement. This is highly dependent on the reactor physics. Outside of the core region the neutron flux decreases substantially by up to more than three orders of magnitude, so radiation damage is less significant outside of the critical region.

\subsubsection{Ingrowth of precipitates}

American Society of Mechanical Engineers (ASME) is currently working on code rules for clad alloys. Molybdenum is a possible choice as a clad material and is known to form intermetallic compounds with Ni. The reaction kinetics are dependent on temperature. The reaction rates are very slow at $700{ }^{\circ} \mathrm{C}$ but occur much more rapidly around $900{ }^{\circ} \mathrm{C}$. The neutron fluence and speed may impact the kinetics. It is important to develop the capability to model solid state precipitation and growth for predicting the impact on structural mechanics.

\subsubsection{Swelling and coarsening}

Microstructure evolution from thermal cycling will have an impact on structural alloy performance. Load following is becoming more important in a grid with progressively larger amounts of non-dispatchable power sources. MSRs can change their input temperature to change the power output. Temperature cycling damages the structural materials. We lack design guidance on the material lifetime impact of temperature cycling. For example, how many cycles are tolerable, how rapid, how deep can they be. Most other reactors are not expected to thermally cycle their components as part of operations, but knowing how much MSRs can employ this strategy is of value.

\section{MSR problems}

Modeling and simulation support industry efforts to design, construct, and operate advanced reactors. The Nuclear Regulatory Commission (NRC) relies upon computational tools to perform assessments related to licensing. Predicting the temporal and spatial distribution of important radioisotopes is critical information for understanding component lifetime, reactor performance, and serves as initial conditions for transient analyses in systems level codes like SAM and severe accident analyses, e.g. using MELCOR. These are used to evaluate the amount of radionuclide release for each design basis accident sequence with conservative estimates of the fuel salt properties derived from the measured data. These results can be used to improve the safety characteristics of the design. Finally, the M\&S toolset provides an understanding on the impact of uncertainty levels for the fuel salt property data on MSR thermal hydraulic, reactor physics, and radionuclide release response relevant to normal operations as well as transient and severe accident scenarios.

As pointed out in the introduction, the overall capability needs in terms of safety adequacy assessment is to develop reasonable, conservative estimates of the quantities, species, and forms of radioactive materials that may be released from MSRs as the result of accidents to support the needs of both the plant owner/operator and the safety regulator but at the same time, not unrealistically conservative so as to significantly impact overall plant costs. This is important to point out because the multi-physics, multi- 
scale capability proposed herein will inevitably include estimated values for many adjustable parameters. The codes will need validation and verification $(V \& V)$. The $V \& V$ will be difficult or impossible to perform for some of the phenomenon as a separate effect and without an operating design specific MSR. A good example is noble metal precipitation and plate out as these products are likely born homogenously in the salt loop on an atomic scale as would also be the case for noble gas nucleation and bubble growth. However, the modeling of each specific plant safety feature, e.g. heat exchangers, off gas system, primary loop, etc., could be validated through a combination of analysis, testing, and experience over a range of conditions that includes normal operations, transient conditions, and design basis accident sequences.

\section{Summary and conclusions}

The objective of the integration roadmap is to describe the necessary physics required for predicting mass accountancy in molten salt reactors. There are a suite of existing DOE codes and new ones, e.g. Yellowjacket-GEM, Yellowjacket-PF, Mole, and MOSCATO, that can be further developed and used to model the physics identified herein. The thermophysical properties and the underlying thermodynamics are fundamental inputs to mass accountancy. The development of the MSTDB results in an integral modeling approach that requires spanning length scales from first principles calculations to the engineering scale. The intention is to predict where material accumulates in a reactor core and loop, to understand perturbations on a systems level, for example the downstream effects from a turbine failure, and to model the behavior and performance of structural materials in contact with molten salt in an MSR. 


\section{REFERENCES}

[1] L. L. Humphries, B. A. Beeny, F. Gelbard, D. L. Louie and J. Phillips, "MELCOR Computer Code Manuals," Sandia National Laboratories, Albuquerque, 2007.

[2] M. R. Tonks, D. Gaston, P. C. Millett, D. Andrs and P. Talbot, "An object-oriented finite element framework for multiphysics phase field simulations," Computational Materials Science, vol. 51, no. 1, pp. 20-29, 2012.

[3] D. Schwen, L. K. Aagesen, J. W. Peterson and M. R. Tonks, "Rapid multiphase-field model development using a modular free energy based approach with automatic differentiation in MOOSE/MARMOT," Computational Materials Science, vol. 132, no. 1, pp. 36-45, 2017.

[4] R. Hu, "A fully-implicit high-order system thermal-hydraulics model for advanced non-LWR safety analyses," Annals of Nuclear Energy, vol. 101, pp. 174-181, 2017.

[5] R. Hu, L. Zou, G. Hu, D. Nunez, T. Mui and T. Fei, "SAM Theory Manual," Argonne National Lab., Argonne, 2021.

[6] B. W. Spencer, W. M. Hoffman, S. Biswas, W. Jiang, A. Giorla and M. A. Backman, "Grizzly and BlackBear: Structural Component Aging Simulation Codes," Nuclear Technology, vol. 207, no. 7, pp. 1-23, 2021.

[7] P. N. Haubenreich and J. R. Engel, "Experience with the Molten-Salt Reactor Experiment," Nuclear Applications and Technology, vol. 8, no. 2, pp. 118-136, 1970.

[8] M. Taube and W. Heer, "Reactor with very low fission product inventroy," Swiss Federal Institute of Reactor Technology, Wurenlingen, 1980.

[9] B. R. Betzler, K. B. Bekar, W. Wieselquist, S. W. Hart and S. G. Stimpson, "Molten Salt Reactor Fuel Depletion Tools in SCALE," in Global/Top Fuel, Seattle, 2019.

[10] B. R. Betzler, R. Heidet, B. Feng, C. Rabiti, T. Sofu and N. R. Brown, "Modeling and simulation functional needs for molten salt reactor licensing," Nuclear Engineering and Design, vol. 355, no. C, 2019.

[11] B. R. Betzler, J. J. Powers, N. R. Brown and B. T. Rearden, "Implementation of Molten Salt Reactor Tools in SCALE," in International Coference on Mathematics and Computational Methods Applied to Nuclear Science and Engineering, Jeju, 2017.

[12] P. W. Humrickhouse and T. F. Fuerst, "Tritium Transport Phenomena in Molten-Salt Reactors," Idaho National Lab., Idaho Falls, 2020.

[13] B. T. Rearden and M. A. Jessee, "Scale Code System 6.2.1," Oak Ridge National Lab., Oak Ridge, 2016. 
[14] J. W. Mcmurray, K. Johnson, C. Agca, B. R. betzler, D. J. Kropaczek, T. M. Besmann, D. Andersson and N. D. B. Ezell, "Roadmap for thermal property measurements of Molten Salt Reactor systems," Oak Ridge National Lab., Oak Ridge, 2021.

[15] A. Pelton and P. Chartrand, "The modified quasi-chemical model: Part II. Multicomponent solutions," Metallurgical and Materials Transactions A, vol. 32, pp. 1355 - 1360, 2001.

[16] U. R. Kattner, "The thermodynamic modeling of multicomponent phase equilibria," JOM, vol. 49, pp. 14-19, 1997.

[17] C.-A. Hwang, J. C. Holste, K. R. Hall and G. A. Mansoori, "A simple relation to predict or to correlate the excess functions of multicomponent mixtures," Fluid Phase Equilibria, vol. 62, no. 3, pp. 173189, 1991.

[18] O. Redlich and A. T. Kister, "Algebraic Representation of Thermodynamic Properties and the Classification of Solutions," Industrial and Engineering Chemistry, vol. 40, no. 2, pp. 345-348, 1948.

[19] G. N. Dul'nev and Y. Zarichnayak, "Thermal conductivity of liquid mixtures," Journal of Engineering physics, vol. 11, no. 6, pp. 400-402, 1996.

[20] A. R. Mahjan and S. R. Mirgane, "Excess Molar Volumes and Viscosities for the Binary Mixtures of n-Octane, n-Decane, n-Dodecane, and n-Tetradecane with Octan-2-ol at $298.15 \mathrm{~K}$," Journal of Thermodynamics, vol. 2013, pp. 1-11, 2013.

[21] R. A. McAllister, "The viscosity of liquid mixtures," AIChE, vol. 6, no. 3, pp. 421-431, 1960.

[22] "CALPHAD Methodology," ThermoCalc, 2021. [Online]. Available: https://thermocalc.com/aboutus/methodology/the-calphad-methodology/.

[23] T. M. Besmann, J. Ard, S. Utlak, J. W. McMurray and R. A. Lefebvre, "Status of the Salt Thermochemical Database," Oak Ridge National Lab., Oak Ridge, 2019.

[24] P. Bajpai, M. Poschmann, D. Andrs, C. Bhave, M. Tonks and M. Piro, "Development of a New Thermochemistry Solver for Multiphysics Simulations of Nuclear Materials," in TMS 149th Annual Meeting \& Exhibition Supplemental Proceedings, 2020.

[25] N. Hoyt and H. Yuan, "Multicomponent Mass Transfer of Actinides in Nuclear Relevant Molten Salts," in Global/TopFuel, Seattle, 2019.

[26] R. Kedl, "Migration of a Class of Fission Products (Noble Metals) in the Molten Salt Reactor Experiment," Oak Ridge National Lab., Oak Ridge, 1972.

[27] J. R. Engel and R. C. Steffy, "Xenon Behavior in the Molten Salt Reactor Experiment," Oak Ridge National Lab., Oak Rdige, 1971.

[28] W. R. Grimes, "CHEMICAL RESEARCH AND DEVELOPMENT FOR MOLTEN-SALT BREEDER REACTORS," Oak Ridge National Lab., Oak Ridge, 1967. 
[29] J. J. Lee, J. D. Arrequi-Mena, C. I. Contescu, T. D. Burchell, Y. Katoh and S. K. Loyalka, "Protection of graphite from salt and gas permeation in molten salt reactors," Journal of Nuclear Materials, vol. 534, 2020.

[30] L. Q. Chen, "Phase-Field Models for Microstructure Evolution," Annual Review of Materials Research, vol. 32, pp. 113-140, 2002.

[31] N. Moelans, B. Blanpain and P. Wollants, "An Introduction to Phase-Field Modeling of Microstructure Evolution," CALPHAD, vol. 32, no. 2, pp. 268-294, 2008.

[32] M. R. Tonks and L. K. Aagesen, "The Phase Field Method: Mesoscale Simulation Aiding Material Discovery," Annual Review of Materials Research, vol. 49, pp. 79-102, 2019.

[33] J. R. Keiser, "Compatibility studies of potential molten-salt breeder reactor materials in molten fluoride salts. [Inconel 601, Cr and Nb modifications of Hastelloy N]," Oak Ridge National Lab., Oak Ridge, 1977.

[34] J. R. Keiser, "Status of tellurium--hastelloy N studies in molten fluoride salts," Oak Ridge National Lab., Oak Ridge, 1977.

[35] H. E. McCoy Jr, " Status of materials development for molten salt reactors," Oak Ridge National Lab., Oak Ridge, 1978. 


APPENDIX A. BURNUP SIMULATION RESULTS 


\begin{tabular}{|c|c|c|c|c|}
\hline \multirow[b]{2}{*}{ Constituent } & \multirow[b]{2}{*}{$\mathbf{t}_{1 / 2}$} & \multicolumn{3}{|c|}{ Composition (mol\%) } \\
\hline & & 1st Year & 10th Year & 30th Year \\
\hline $\mathbf{F}$ & & $\mathbf{5 9 . 4 7 7}$ & 59.452 & 59.394 \\
\hline${ }^{19} \mathrm{~F}$ & Stable & 59.477 & 59.452 & 59.394 \\
\hline $\mathbf{L i}$ & & 26.338 & 26.327 & 26.296 \\
\hline${ }^{7} \mathrm{Li}$ & Stable & 26.338 & 26.327 & 26.296 \\
\hline Be & & 11.791 & 11.786 & 11.774 \\
\hline${ }^{9} \mathrm{Be}$ & Stable & 11.791 & 11.786 & 11.774 \\
\hline $\mathbf{Z r}$ & & 2.025 & 2.036 & 2.059 \\
\hline${ }^{90} \mathrm{Zr}$ & Stable & 1.041 & 1.041 & 1.042 \\
\hline${ }^{94} \mathrm{Zr}$ & Stable & 0.352 & 0.354 & 0.359 \\
\hline${ }^{92} \mathrm{Zr}$ & Stable & 0.347 & 0.350 & 0.355 \\
\hline${ }^{91} \mathrm{Zr}$ & Stable & 0.227 & 0.229 & 0.231 \\
\hline${ }^{96} \mathrm{Zr}$ & Stable & 0.057 & 0.059 & 0.064 \\
\hline${ }^{93} \mathrm{Zr}$ & $1.6 \times 10^{6} \mathrm{yrs}$ & 0.000 & 0.003 & 0.008 \\
\hline $\mathbf{U}$ & & 0.360 & 0.316 & 0.226 \\
\hline${ }^{238} \mathrm{U}$ & $4.5 \times 10^{9} \mathrm{yrs}$ & 0.236 & 0.229 & 0.205 \\
\hline${ }^{235} \mathrm{U}$ & $7.0 \times 10^{8} \mathrm{yrs}$ & 0.122 & 0.078 & 0.002 \\
\hline${ }^{236} \mathrm{U}$ & $2.3 \times 10^{7} \mathrm{yrs}$ & 0.001 & 0.009 & 0.019 \\
\hline Mo & & 0.001 & 0.011 & 0.032 \\
\hline${ }^{100} \mathrm{Mo}$ & $7.3 \times 10^{18} \mathrm{yrs}$ & 0.000 & 0.003 & 0.008 \\
\hline${ }^{95} \mathrm{Mo}$ & Stable & 0.000 & 0.003 & 0.008 \\
\hline${ }^{97} \mathrm{Mo}$ & Stable & 0.000 & 0.003 & 0.008 \\
\hline${ }^{98} \mathrm{Mo}$ & Stable & 0.000 & 0.003 & 0.007 \\
\hline $\mathrm{Xe}$ & & 0.001 & 0.009 & 0.028 \\
\hline${ }^{134} \mathrm{Xe}$ & Stable & 0.000 & 0.003 & 0.010 \\
\hline${ }^{136} \mathrm{Xe}$ & Stable & 0.000 & 0.003 & 0.008 \\
\hline${ }^{132} \mathrm{Xe}$ & Stable & 0.000 & 0.002 & 0.006 \\
\hline${ }^{131} \mathrm{Xe}$ & Stable & 0.000 & 0.001 & 0.004 \\
\hline Nd & & 0.001 & 0.009 & 0.025 \\
\hline${ }^{144} \mathrm{Nd}$ & $2.3 \times 10^{15} \mathrm{yrs}$ & 0.000 & 0.002 & 0.012 \\
\hline${ }^{143} \mathrm{Nd}$ & Stable & 0.000 & 0.002 & 0.002 \\
\hline${ }^{146} \mathrm{Nd}$ & Stable & 0.000 & 0.001 & 0.005 \\
\hline${ }^{145} \mathrm{Nd}$ & Stable & 0.000 & 0.002 & 0.004 \\
\hline${ }^{148} \mathrm{Nd}$ & Stable & 0.000 & 0.001 & 0.002 \\
\hline${ }^{150} \mathrm{Nd}$ & Stable & 0.000 & 0.000 & 0.001 \\
\hline Cs & & 0.001 & 0.008 & 0.024 \\
\hline${ }^{135} \mathrm{Cs}$ & $1.3 \times 10^{6} \mathrm{yrs}$ & 0.000 & 0.003 & 0.009 \\
\hline${ }^{133} \mathrm{Cs}$ & Stable & 0.000 & 0.003 & 0.009 \\
\hline${ }^{137} \mathrm{Cs}$ & $30.1 \mathrm{yrs}$ & 0.000 & 0.002 & 0.006 \\
\hline
\end{tabular}




\begin{tabular}{|c|c|c|c|c|}
\hline He & & 0.000 & 0.003 & 0.020 \\
\hline${ }^{4} \mathrm{He}$ & Stable & 0.000 & 0.003 & 0.020 \\
\hline Ru & & 0.000 & 0.002 & 0.007 \\
\hline${ }^{101} \mathrm{Ru}$ & Stable & 0.000 & 0.002 & 0.007 \\
\hline${ }^{102} \mathrm{Ru}$ & Stable & 0.000 & 0.002 & 0.006 \\
\hline${ }^{104} \mathrm{Ru}$ & Stable & 0.000 & 0.001 & 0.004 \\
\hline $\mathrm{Ce}$ & & 0.001 & 0.005 & 0.015 \\
\hline${ }^{140} \mathrm{Ce}$ & Stable & 0.000 & 0.003 & 0.008 \\
\hline${ }^{142} \mathrm{Ce}$ & Stable & 0.000 & 0.003 & 0.007 \\
\hline $\mathbf{B a}$ & & 0.000 & 0.003 & 0.011 \\
\hline${ }^{137} \mathrm{Ba}$ & Stable & 0.000 & 0.003 & 0.009 \\
\hline${ }^{138} \mathrm{Ba}$ & Stable & 0.000 & 0.000 & 0.002 \\
\hline $\mathbf{S r}$ & & 0.000 & 0.004 & 0.008 \\
\hline${ }^{90} \mathrm{Sr}$ & $28.8 \mathrm{yrs}$ & 0.000 & 0.002 & 0.005 \\
\hline${ }^{88} \mathrm{Sr}$ & Stable & 0.000 & 0.001 & 0.004 \\
\hline Te & & 0.000 & 0.003 & 0.008 \\
\hline${ }^{99} \mathrm{Tc}$ & $2.1 \times 10^{5} \mathrm{yrs}$ & 0.000 & 0.003 & 0.008 \\
\hline La & & 0.000 & 0.003 & 0.008 \\
\hline${ }^{139} \mathrm{La}$ & Stable & 0.000 & 0.003 & 0.008 \\
\hline Pr & & 0.000 & 0.002 & 0.007 \\
\hline${ }^{141} \operatorname{Pr}$ & Stable & 0.000 & 0.002 & 0.007 \\
\hline Pd & & 0.000 & 0.001 & 0.006 \\
\hline${ }^{105} \mathrm{Pd}$ & Stable & 0.000 & 0.001 & 0.003 \\
\hline${ }^{106} \mathrm{Pd}$ & Stable & 0.000 & 0.000 & 0.002 \\
\hline${ }^{107} \mathrm{Pd}$ & $6.5 \times 10^{6} \mathrm{yrs}$ & 0.000 & 0.000 & 0.001 \\
\hline${ }^{108} \mathrm{Pd}$ & Stable & 0.000 & 0.000 & 0.001 \\
\hline $\mathbf{P u}$ & & 0.001 & 0.004 & 0.005 \\
\hline${ }^{239} \mathrm{Pu}$ & $2.4 \times 10^{4} \mathrm{yrs}$ & 0.001 & 0.003 & 0.001 \\
\hline${ }^{240} \mathrm{Pu}$ & $6561 \mathrm{yrs}$ & 0.000 & 0.001 & 0.001 \\
\hline${ }^{242} \mathrm{Pu}$ & $3.8 \times 10^{5} \mathrm{yrs}$ & 0.000 & 0.000 & 0.002 \\
\hline${ }^{238} \mathrm{Pu}$ & $87.7 \mathrm{yrs}$ & 0.000 & 0.000 & 0.001 \\
\hline $\mathbf{Y}$ & & 0.000 & 0.002 & 0.005 \\
\hline${ }^{89} \mathrm{Y}$ & Stable & 0.000 & 0.002 & 0.005 \\
\hline I & & 0.000 & 0.000 & 0.001 \\
\hline${ }^{129} \mathrm{I}$ & $1.6 \times 10^{7} \mathrm{yrs}$ & 0.000 & 0.000 & 0.001 \\
\hline
\end{tabular}


MSRE - By Stream

\begin{tabular}{|c|c|c|c|c|c|}
\hline \multirow[b]{2}{*}{ Constituent } & \multicolumn{5}{|c|}{ Composition (mol\%) } \\
\hline & $\mathbf{t}_{1 / 2}$ & 1st Year & 10th Year & 30th Year & Stream \\
\hline $\mathbf{F}$ & & 59.477 & $\mathbf{5 9 . 4 5 2}$ & 59.394 & \\
\hline${ }^{19} \mathrm{~F}$ & Stable & 59.477 & 59.452 & 59.394 & Fuel \\
\hline $\mathbf{L i}$ & & 26.338 & 26.327 & 26.296 & \\
\hline${ }^{7} \mathrm{Li}$ & Stable & 26.338 & 26.327 & 26.296 & Fuel \\
\hline $\mathbf{B e}$ & & 11.791 & 11.786 & 11.774 & \\
\hline${ }^{9} \mathrm{Be}$ & Stable & 11.791 & 11.786 & 11.774 & Fuel \\
\hline $\mathbf{Z r}$ & & 2.025 & 2.036 & 2.059 & \\
\hline${ }^{90} \mathrm{Zr}$ & Stable & 1.041 & 1.041 & 1.042 & Fuel \\
\hline${ }^{94} \mathrm{Zr}$ & Stable & 0.352 & 0.354 & 0.359 & Fuel \\
\hline${ }^{92} \mathrm{Zr}$ & Stable & 0.347 & 0.350 & 0.355 & Fuel \\
\hline${ }^{91} \mathrm{Zr}$ & Stable & 0.227 & 0.229 & 0.231 & Fuel \\
\hline${ }^{96} \mathrm{Zr}$ & Stable & 0.057 & 0.059 & 0.064 & Fuel \\
\hline${ }^{93} \mathrm{Zr}$ & $1.6 \times 10^{6} \mathrm{yrs}$ & 0.000 & 0.003 & 0.008 & Fuel \\
\hline $\mathbf{U}$ & & 0.360 & 0.316 & 0.226 & \\
\hline${ }^{238} \mathrm{U}$ & $4.5 \times 10^{9} \mathrm{yrs}$ & 0.236 & 0.229 & 0.205 & Fuel \\
\hline${ }^{235} \mathrm{U}$ & $7.0 \times 10^{8} \mathrm{yrs}$ & 0.001 & 0.009 & 0.019 & Fuel \\
\hline${ }^{236} \mathrm{U}$ & $2.3 \times 10^{7} \mathrm{yrs}$ & 0.122 & 0.078 & 0.002 & Fuel \\
\hline Mo & & 0.001 & 0.011 & 0.032 & \\
\hline${ }^{100} \mathrm{Mo}$ & $7.3 \times 10^{18} \mathrm{yrs}$ & 0.000 & 0.003 & 0.008 & Solid Waste \\
\hline${ }^{95} \mathrm{Mo}$ & Stable & 0.000 & 0.003 & 0.008 & Solid Waste \\
\hline${ }^{97} \mathrm{Mo}$ & Stable & 0.000 & 0.003 & 0.008 & Solid Waste \\
\hline${ }^{98} \mathrm{Mo}$ & Stable & 0.000 & 0.003 & 0.007 & Solid Waste \\
\hline Nd & & 0.001 & 0.009 & 0.025 & \\
\hline${ }^{144} \mathrm{Nd}$ & $2.3 \times 10^{15} \mathrm{yrs}$ & 0.000 & 0.002 & 0.012 & Fuel \\
\hline${ }^{146} \mathrm{Nd}$ & Stable & 0.000 & 0.001 & 0.005 & Fuel \\
\hline${ }^{145} \mathrm{Nd}$ & Stable & 0.000 & 0.002 & 0.004 & Fuel \\
\hline${ }^{148} \mathrm{Nd}$ & Stable & 0.000 & 0.001 & 0.002 & Fuel \\
\hline${ }^{143} \mathrm{Nd}$ & Stable & 0.000 & 0.002 & 0.002 & Fuel \\
\hline${ }^{150} \mathrm{Nd}$ & Stable & 0.000 & 0.000 & 0.001 & Fuel \\
\hline $\mathbf{X e}$ & & 0.001 & 0.009 & 0.028 & \\
\hline${ }^{134} \mathrm{Xe}$ & Stable & 0.000 & 0.003 & 0.010 & Gas Waste \\
\hline${ }^{136} \mathrm{Xe}$ & Stable & 0.000 & 0.003 & 0.008 & Gas Waste \\
\hline${ }^{132} \mathrm{Xe}$ & Stable & 0.000 & 0.002 & 0.005 & Gas Waste \\
\hline${ }^{131} \mathrm{Xe}$ & Stable & 0.000 & 0.001 & 0.004 & Gas Waste \\
\hline${ }^{132} \mathrm{Xe}$ & Stable & 0.000 & 0.000 & 0.001 & Gas Waste \\
\hline $\mathrm{He}$ & & 0.000 & 0.003 & 0.020 & \\
\hline${ }^{4} \mathrm{He}$ & Stable & 0.000 & 0.003 & 0.020 & Fuel \\
\hline Cs & & 0.001 & 0.008 & 0.023 & \\
\hline
\end{tabular}




\begin{tabular}{|c|c|c|c|c|c|}
\hline${ }^{135} \mathrm{Cs}$ & $1.3 \times 10^{6} \mathrm{yrs}$ & 0.000 & 0.003 & 0.009 & Gas Waste \\
\hline${ }^{133} \mathrm{Cs}$ & Stable & 0.000 & 0.003 & 0.009 & Gas Waste \\
\hline${ }^{137} \mathrm{Cs}$ & $30.1 \mathrm{yrs}$ & 0.000 & 0.002 & 0.006 & Fuel \\
\hline $\mathbf{R u}$ & & 0.000 & 0.005 & 0.017 & \\
\hline${ }^{101} \mathrm{Ru}$ & Stable & 0.000 & 0.002 & 0.007 & Solid Waste \\
\hline${ }^{102} \mathrm{Ru}$ & Stable & 0.000 & 0.002 & 0.006 & Solid Waste \\
\hline${ }^{104} \mathrm{Ru}$ & Stable & 0.000 & 0.001 & 0.004 & Solid Waste \\
\hline $\mathrm{Ce}$ & & 0.001 & 0.005 & 0.015 & \\
\hline${ }^{140} \mathrm{Ce}$ & Stable & 0.000 & 0.003 & 0.008 & Fuel \\
\hline${ }^{142} \mathrm{Ce}$ & Stable & 0.000 & 0.003 & 0.007 & Fuel \\
\hline Ba & & 0.000 & 0.003 & 0.011 & \\
\hline${ }^{138} \mathrm{Ba}$ & Stable & 0.000 & 0.003 & 0.009 & Fuel \\
\hline${ }^{137} \mathrm{Ba}$ & Stable & 0.000 & 0.000 & 0.002 & Fuel \\
\hline Sr & & 0.000 & 0.004 & 0.008 & \\
\hline${ }^{90} \mathrm{Sr}$ & $28.8 \mathrm{yrs}$ & 0.000 & 0.002 & 0.005 & Fuel \\
\hline${ }^{88} \mathrm{Sr}$ & Stable & 0.000 & 0.001 & 0.004 & Fuel \\
\hline Te & & 0.000 & 0.003 & 0.008 & \\
\hline${ }^{99} \mathrm{Tc}$ & $2.1 \times 10^{5} \mathrm{yrs}$ & 0.000 & 0.003 & 0.008 & Solid Waste \\
\hline La & & 0.000 & 0.003 & 0.008 & \\
\hline${ }^{139} \mathrm{La}$ & Stable & 0.000 & 0.003 & 0.008 & Fuel \\
\hline Pr & & 0.000 & 0.002 & 0.007 & \\
\hline${ }^{141} \operatorname{Pr}$ & Stable & 0.000 & 0.002 & 0.007 & Fuel \\
\hline Pd & & 0.000 & 0.001 & 0.006 & \\
\hline${ }^{105} \mathrm{Pd}$ & Stable & 0.000 & 0.001 & 0.003 & Solid Waste \\
\hline${ }^{106} \mathrm{Pd}$ & Stable & 0.000 & 0.000 & 0.002 & Solid Waste \\
\hline${ }^{107} \mathrm{Pd}$ & $6.5 \times 10^{6} \mathrm{yrs}$ & 0.000 & 0.000 & 0.001 & Solid Waste \\
\hline${ }^{108} \mathrm{Pd}$ & Stable & 0.000 & 0.000 & 0.001 & Solid Waste \\
\hline $\mathbf{P u}$ & & 0.001 & 0.004 & 0.005 & \\
\hline${ }^{242} \mathrm{Pu}$ & $3.8 \times 10^{5} \mathrm{yrs}$ & 0.000 & 0.000 & 0.002 & Fuel \\
\hline${ }^{240} \mathrm{Pu}$ & $6561 \mathrm{yrs}$ & 0.000 & 0.001 & 0.001 & Fuel \\
\hline${ }^{238} \mathrm{Pu}$ & 87.7 yrs & 0.000 & 0.000 & 0.001 & Fuel \\
\hline${ }^{239} \mathrm{Pu}$ & $2.4 \times 10^{4} \mathrm{yrs}$ & 0.001 & 0.003 & 0.001 & Fuel \\
\hline I & & 0.000 & 0.000 & 0.001 & \\
\hline${ }^{129} \mathrm{I}$ & $1.6 \times 10^{7} \mathrm{yrs}$ & 0.000 & 0.000 & 0.001 & Fuel \\
\hline
\end{tabular}


SOFT - Total

\begin{tabular}{|c|c|c|c|c|}
\hline \multirow[b]{2}{*}{ Constituent } & \multirow[b]{2}{*}{$\mathbf{t}_{1 / 2}$} & \multicolumn{3}{|c|}{ Composition (mol\%) } \\
\hline & & 1st Year & 10th Year & 30th Year \\
\hline Cl & & 65.960 & 64.940 & 62.760 \\
\hline${ }^{37} \mathrm{Cl}$ & Stable & 65.960 & 64.940 & 62.760 \\
\hline $\mathbf{N a}$ & & 17.817 & 17.533 & 16.950 \\
\hline${ }^{23} \mathrm{Na}$ & Stable & 17.817 & 17.533 & 16.950 \\
\hline $\mathbf{U}$ & & 14.244 & 13.872 & 13.195 \\
\hline${ }^{234} \mathrm{U}$ & $2.5 \times 10^{5} \mathrm{yrs}$ & 0.000 & 0.000 & 0.001 \\
\hline${ }^{235} \mathrm{U}$ & $7.0 \times 10^{8} \mathrm{yrs}$ & 0.097 & 0.058 & 0.021 \\
\hline${ }^{236} \mathrm{U}$ & $2.3 \times 10^{7} \mathrm{yrs}$ & 0.001 & 0.009 & 0.015 \\
\hline${ }^{238} \mathrm{U}$ & $4.5 \times 10^{9} \mathrm{yrs}$ & 14.146 & 13.804 & 13.158 \\
\hline $\mathbf{P u}$ & & 1.797 & 1.882 & 2.001 \\
\hline${ }^{238} \mathrm{Pu}$ & 87.7 yrs & 0.000 & 0.004 & 0.014 \\
\hline${ }^{239} \mathrm{Pu}$ & $2.4 \times 10^{4} \mathrm{yrs}$ & 1.350 & 1.415 & 1.440 \\
\hline${ }^{240} \mathrm{Pu}$ & $6500 \mathrm{yrs}$ & 0.188 & 0.265 & 0.395 \\
\hline${ }^{241} \mathrm{Pu}$ & $14 \mathrm{yrs}$ & 0.081 & 0.042 & 0.034 \\
\hline${ }^{242} \mathrm{Pu}$ & $3.7 \times 10^{5} \mathrm{yrs}$ & 0.177 & 0.155 & 0.117 \\
\hline $\mathrm{Xe}$ & & 0.020 & 0.198 & 0.576 \\
\hline${ }^{130} \mathrm{Xe}$ & Stable & 0.000 & 0.000 & 0.003 \\
\hline${ }^{131} \mathrm{Xe}$ & Stable & 0.003 & 0.028 & 0.082 \\
\hline${ }^{132} \mathrm{Xe}$ & Stable & 0.004 & 0.044 & 0.128 \\
\hline${ }^{134} \mathrm{Xe}$ & Stable & 0.007 & 0.065 & 0.189 \\
\hline${ }^{136} \mathrm{Xe}$ & $2.2 \times 10^{21} \mathrm{yrs}$ & 0.006 & 0.060 & 0.174 \\
\hline Мo & & 0.018 & 0.191 & 0.559 \\
\hline${ }^{95} \mathrm{Mo}$ & Stable & 0.003 & 0.040 & 0.118 \\
\hline${ }^{97} \mathrm{Mo}$ & Stable & 0.005 & 0.045 & 0.132 \\
\hline${ }^{98} \mathrm{Mo}$ & Stable & 0.005 & 0.049 & 0.143 \\
\hline${ }^{100} \mathrm{Mo}$ & $7.8 \times 10^{18} \mathrm{yrs}$ & 0.006 & 0.057 & 0.166 \\
\hline $\mathbf{Z r}$ & & 0.018 & 0.171 & 0.500 \\
\hline${ }^{90} \mathrm{Zr}$ & Stable & 0.000 & 0.002 & 0.017 \\
\hline${ }^{91} \mathrm{Zr}$ & Stable & 0.002 & 0.024 & 0.068 \\
\hline${ }^{92} \mathrm{Zr}$ & Stable & 0.003 & 0.028 & 0.081 \\
\hline${ }^{93} \mathrm{Zr}$ & $1.5 \times 10^{6} \mathrm{yrs}$ & 0.003 & 0.034 & 0.099 \\
\hline${ }^{94} \mathrm{Zr}$ & Stable & 0.004 & 0.037 & 0.108 \\
\hline${ }^{95} \mathrm{Zr}$ & 64.0 days & 0.001 & 0.001 & 0.001 \\
\hline${ }^{96} \mathrm{Zr}$ & $2.0 \times 10^{19} \mathrm{yrs}$ & 0.004 & 0.044 & 0.126 \\
\hline Cs & & 0.018 & 0.178 & 0.489 \\
\hline${ }^{133} \mathrm{Cs}$ & Stable & 0.006 & 0.056 & 0.162 \\
\hline${ }^{135} \mathrm{Cs}$ & $2.3 \times 10^{6} \mathrm{yrs}$ & 0.007 & 0.072 & 0.211 \\
\hline${ }^{137} \mathrm{Cs}$ & 30.2 yrs & 0.006 & 0.050 & 0.117 \\
\hline $\mathbf{R u}$ & & 0.019 & 0.162 & 0.462 \\
\hline${ }^{101} \mathrm{Ru}$ & Stable & 0.005 & 0.053 & 0.153 \\
\hline${ }^{102} \mathrm{Ru}$ & Stable & 0.005 & 0.053 & 0.155 \\
\hline${ }^{103} \mathrm{Ru}$ & 39.3 days & 0.001 & 0.001 & 0.001 \\
\hline${ }^{104} \mathrm{Ru}$ & Stable & 0.005 & 0.050 & 0.148 \\
\hline${ }^{106} \mathrm{Ru}$ & 373.6 days & 0.003 & 0.005 & 0.005 \\
\hline Nd & & 0.012 & 0.142 & 0.418 \\
\hline${ }^{143} \mathrm{Nd}$ & Stable & 0.004 & 0.038 & 0.111 \\
\hline${ }^{144} \mathrm{Nd}$ & $2.3 \times 10^{15} \mathrm{yrs}$ & 0.001 & 0.030 & 0.093 \\
\hline
\end{tabular}




\begin{tabular}{|c|c|c|c|c|}
\hline${ }^{145} \mathrm{Nd}$ & Stable & 0.003 & 0.027 & 0.078 \\
\hline${ }^{146} \mathrm{Nd}$ & Stable & 0.002 & 0.023 & 0.066 \\
\hline${ }^{148} \mathrm{Nd}$ & Stable & 0.002 & 0.015 & 0.043 \\
\hline${ }^{150} \mathrm{Nd}$ & $6.7 \times 10^{18} \mathrm{yrs}$ & 0.001 & 0.009 & 0.026 \\
\hline Pd & & 0.010 & 0.124 & 0.374 \\
\hline${ }^{105} \mathrm{Pd}$ & Stable & 0.005 & 0.047 & 0.137 \\
\hline${ }^{106} \mathrm{Pd}$ & Stable & 0.001 & 0.029 & 0.096 \\
\hline${ }^{107} \mathrm{Pd}$ & $6.5 \times 10^{6} \mathrm{yrs}$ & 0.003 & 0.026 & 0.076 \\
\hline${ }^{108} \mathrm{Pd}$ & Stable & 0.002 & 0.017 & 0.049 \\
\hline${ }^{110} \mathrm{Pd}$ & Stable & 0.001 & 0.005 & 0.016 \\
\hline $\mathrm{Ce}$ & & 0.012 & 0.093 & 0.262 \\
\hline${ }^{140} \mathrm{Ce}$ & Stable & 0.005 & 0.047 & 0.137 \\
\hline${ }^{141} \mathrm{Ce}$ & 32.5 days & 0.001 & 0.001 & 0.001 \\
\hline${ }^{142} \mathrm{Ce}$ & - & 0.004 & 0.042 & 0.121 \\
\hline${ }^{144} \mathrm{Ce}$ & 284.9 days & 0.002 & 0.004 & 0.004 \\
\hline $\mathbf{B a}$ & & 0.006 & 0.063 & 0.209 \\
\hline${ }^{136} \mathrm{Ba}$ & Stable & 0.000 & 0.001 & 0.002 \\
\hline${ }^{137} \mathrm{Ba}$ & Stable & 0.000 & 0.006 & 0.045 \\
\hline${ }^{138} \mathrm{Ba}$ & Stable & 0.006 & 0.056 & 0.162 \\
\hline Rh & & 0.005 & $\mathbf{0 . 0 5 5}$ & 0.164 \\
\hline${ }^{103} \mathrm{Rh}$ & Stable & 0.005 & 0.055 & 0.164 \\
\hline Tc & & 0.005 & $\mathbf{0 . 0 5 0}$ & 0.145 \\
\hline${ }^{99} \mathrm{Tc}$ & $2.1 \times 10^{5} \mathrm{yrs}$ & 0.005 & 0.050 & 0.145 \\
\hline La & & 0.005 & 0.049 & 0.142 \\
\hline${ }^{139} \mathrm{La}$ & Stable & 0.005 & 0.049 & 0.142 \\
\hline Pr & & 0.004 & 0.044 & 0.130 \\
\hline${ }^{141} \operatorname{Pr}$ & Stable & 0.004 & 0.044 & 0.130 \\
\hline Sm & & 0.003 & 0.036 & 0.116 \\
\hline${ }^{147} \mathrm{Sm}$ & $1.1 \times 10^{11} \mathrm{yrs}$ & 0.000 & 0.012 & 0.046 \\
\hline${ }^{149} \mathrm{Sm}$ & Stable & 0.001 & 0.011 & 0.032 \\
\hline${ }^{151} \mathrm{Sm}$ & $88.8 \mathrm{yrs}$ & 0.001 & 0.006 & 0.017 \\
\hline${ }^{152} \mathrm{Sm}$ & Stable & 0.000 & 0.005 & 0.014 \\
\hline${ }^{154} \mathrm{Sm}$ & Stable & 0.000 & 0.002 & 0.006 \\
\hline Sr & & 0.003 & $\mathbf{0 . 0 3 0}$ & 0.076 \\
\hline${ }^{88} \mathrm{Sr}$ & Stable & 0.001 & 0.012 & 0.035 \\
\hline${ }^{90} \mathrm{Sr}$ & $28.9 \mathrm{yrs}$ & 0.002 & 0.018 & 0.041 \\
\hline Te & & 0.003 & 0.026 & 0.075 \\
\hline${ }^{125} \mathrm{Te}$ & Stable & 0.000 & 0.000 & 0.002 \\
\hline${ }^{128} \mathrm{Te}$ & $2.2 \times 10^{24} \mathrm{yrs}$ & 0.001 & 0.006 & 0.017 \\
\hline${ }^{130} \mathrm{Te}$ & $8.2 \times 10^{20} \mathrm{yrs}$ & 0.002 & 0.019 & 0.056 \\
\hline $\mathbf{I}$ & & 0.001 & 0.011 & 0.030 \\
\hline${ }^{127} \mathrm{I}$ & Stable & 0.000 & 0.001 & 0.003 \\
\hline${ }^{129} \mathrm{I}$ & $1.6 \times 10^{7} \mathrm{yrs}$ & 0.001 & 0.010 & 0.027 \\
\hline
\end{tabular}




\begin{tabular}{|c|c|c|c|c|c|}
\hline \multirow[b]{2}{*}{ Constituent } & \multicolumn{5}{|c|}{ Composition (mol\%) } \\
\hline & $\mathbf{t}_{1 / 2}$ & 1st Year & 10th Year & 30th Year & Stream \\
\hline $\mathbf{C l}$ & & 65.961 & 64.943 & 62.767 & \\
\hline${ }^{37} \mathrm{Cl}$ & Stable & 65.961 & 64.943 & 62.767 & Fuel \\
\hline $\mathbf{N a}$ & & 17.817 & $\mathbf{1 7 . 5 3 3}$ & 16.950 & \\
\hline${ }^{23} \mathrm{Na}$ & Stable & 17.817 & 17.533 & 16.950 & Fuel \\
\hline $\mathbf{U}$ & & 14.244 & 13.872 & 13.195 & \\
\hline${ }^{234} \mathrm{U}$ & $2.5 \times 10^{5} \mathrm{yrs}$ & 0.000 & 0.000 & 0.001 & Fuel \\
\hline${ }^{235} \mathrm{U}$ & $7.0 \times 10^{8} \mathrm{yrs}$ & 0.097 & 0.058 & 0.021 & Fuel \\
\hline${ }^{236} \mathrm{U}$ & $2.3 \times 10^{7} \mathrm{yrs}$ & 0.001 & 0.009 & 0.015 & Fuel \\
\hline${ }^{238} \mathrm{U}$ & $4.5 \times 10^{9} \mathrm{yrs}$ & 14.146 & 13.804 & 13.158 & Fuel \\
\hline $\mathbf{P u}$ & & 1.797 & 1.882 & 2.001 & \\
\hline${ }^{238} \mathrm{Pu}$ & $87.7 \mathrm{yrs}$ & 0.000 & 0.004 & 0.014 & Fuel \\
\hline${ }^{239} \mathrm{Pu}$ & $2.4 \times 10^{4} \mathrm{yrs}$ & 1.350 & 1.415 & 1.440 & Fuel \\
\hline${ }^{240} \mathrm{Pu}$ & $6500 \mathrm{yrs}$ & 0.188 & 0.265 & 0.395 & Fuel \\
\hline${ }^{241} \mathrm{Pu}$ & 14 yrs & 0.081 & 0.042 & 0.034 & Fuel \\
\hline${ }^{242} \mathrm{Pu}$ & $3.7 \times 10^{5} \mathrm{yrs}$ & 0.177 & 0.155 & 0.117 & Fuel \\
\hline $\mathbf{X e}$ & & 0.020 & 0.198 & 0.576 & \\
\hline${ }^{130} \mathrm{Xe}$ & Stable & 0.000 & 0.000 & 0.003 & Waste \\
\hline${ }^{131} \mathrm{Xe}$ & Stable & 0.003 & 0.028 & 0.082 & Waste \\
\hline${ }^{132} \mathrm{Xe}$ & Stable & 0.004 & 0.044 & 0.128 & Waste \\
\hline${ }^{134} \mathrm{Xe}$ & Stable & 0.007 & 0.065 & 0.189 & Waste \\
\hline${ }^{136} \mathrm{Xe}$ & $2.2 \times 10^{21} \mathrm{yrs}$ & 0.006 & 0.060 & 0.174 & Waste \\
\hline Mo & & 0.018 & 0.191 & 0.559 & \\
\hline${ }^{95} \mathrm{Mo}$ & Stable & 0.003 & 0.040 & 0.118 & Waste \\
\hline${ }^{97} \mathrm{Mo}$ & Stable & 0.005 & 0.045 & 0.132 & Waste \\
\hline${ }^{98} \mathrm{Mo}$ & Stable & 0.005 & 0.049 & 0.143 & Waste \\
\hline${ }^{100} \mathrm{Mo}$ & $7.8 \times 10^{18} \mathrm{yrs}$ & 0.006 & 0.057 & 0.166 & Waste \\
\hline $\mathbf{Z r}$ & & 0.018 & 0.171 & 0.500 & \\
\hline${ }^{90} \mathrm{Zr}$ & Stable & 0.000 & 0.002 & 0.017 & Waste \\
\hline${ }^{91} \mathrm{Zr}$ & Stable & 0.002 & 0.024 & 0.068 & Waste \\
\hline${ }^{92} \mathrm{Zr}$ & Stable & 0.003 & 0.028 & 0.081 & Waste \\
\hline${ }^{93} \mathrm{Zr}$ & $1.5 \times 10^{6} \mathrm{yrs}$ & 0.003 & 0.034 & 0.099 & Waste \\
\hline${ }^{94} \mathrm{Zr}$ & Stable & 0.004 & 0.037 & 0.108 & Waste \\
\hline${ }^{95} \mathrm{Zr}$ & 64.0 days & 0.001 & 0.001 & 0.001 & Waste \\
\hline${ }^{96} \mathrm{Zr}$ & $2.0 \times 10^{19} \mathrm{yrs}$ & 0.004 & 0.044 & 0.126 & Waste \\
\hline Cs & & 0.018 & 0.178 & 0.489 & \\
\hline${ }^{133} \mathrm{Cs}$ & Stable & 0.006 & 0.056 & 0.162 & Waste \\
\hline${ }^{135} \mathrm{Cs}$ & $2.3 \times 10^{6} \mathrm{yrs}$ & 0.007 & 0.072 & 0.211 & Waste \\
\hline${ }^{137} \mathrm{Cs}$ & $30.2 \mathrm{yrs}$ & 0.004 & 0.048 & 0.115 & Waste \\
\hline${ }^{137} \mathrm{Cs}$ & $30.2 \mathrm{yrs}$ & 0.002 & 0.002 & 0.002 & Fuel \\
\hline $\mathbf{R u}$ & & 0.019 & 0.162 & 0.462 & \\
\hline${ }^{101} \mathrm{Ru}$ & Stable & 0.005 & 0.053 & 0.153 & Waste \\
\hline${ }^{102} \mathrm{Ru}$ & Stable & 0.005 & 0.053 & 0.155 & Waste \\
\hline${ }^{103} \mathrm{Ru}$ & 39.3 days & 0.001 & 0.001 & 0.001 & Waste \\
\hline${ }^{104} \mathrm{Ru}$ & Stable & 0.005 & 0.050 & 0.148 & Waste \\
\hline${ }^{106} \mathrm{Ru}$ & 373.6 days & 0.003 & 0.005 & 0.005 & Waste \\
\hline Nd & & 0.012 & 0.142 & 0.418 & \\
\hline${ }^{143} \mathrm{Nd}$ & Stable & 0.004 & 0.038 & 0.112 & Waste \\
\hline${ }^{144} \mathrm{Nd}$ & $2.3 \times 10^{15} \mathrm{yrs}$ & 0.001 & 0.030 & 0.093 & Waste \\
\hline
\end{tabular}




\begin{tabular}{|c|c|c|c|c|c|}
\hline${ }^{145} \mathrm{Nd}$ & Stable & 0.003 & 0.027 & 0.078 & Waste \\
\hline${ }^{146} \mathrm{Nd}$ & Stable & 0.002 & 0.023 & 0.066 & Waste \\
\hline${ }^{148} \mathrm{Nd}$ & Stable & 0.002 & 0.015 & 0.043 & Waste \\
\hline${ }^{150} \mathrm{Nd}$ & $6.7 \times 10^{18} \mathrm{yrs}$ & 0.001 & 0.009 & 0.026 & Waste \\
\hline Pd & & 0.010 & 0.124 & 0.374 & \\
\hline${ }^{105} \mathrm{Pd}$ & Stable & 0.005 & 0.047 & 0.137 & Waste \\
\hline${ }^{106} \mathrm{Pd}$ & Stable & 0.001 & 0.029 & 0.096 & Waste \\
\hline${ }^{107} \mathrm{Pd}$ & $6.5 \times 10^{6} \mathrm{yrs}$ & 0.003 & 0.026 & 0.076 & Waste \\
\hline${ }^{108} \mathrm{Pd}$ & Stable & 0.002 & 0.017 & 0.049 & Waste \\
\hline${ }^{110} \mathrm{Pd}$ & Stable & 0.001 & 0.005 & 0.016 & Waste \\
\hline $\mathrm{Ce}$ & & 0.012 & 0.093 & 0.262 & \\
\hline${ }^{140} \mathrm{Ce}$ & Stable & 0.005 & 0.047 & 0.137 & Waste \\
\hline${ }^{141} \mathrm{Ce}$ & 32.5 days & 0.001 & 0.001 & 0.001 & Waste \\
\hline${ }^{142} \mathrm{Ce}$ & Stable & 0.004 & 0.042 & 0.121 & Waste \\
\hline${ }^{144} \mathrm{Ce}$ & 284.9 days & 0.002 & 0.004 & 0.004 & Waste \\
\hline Ba & & 0.005 & 0.052 & 0.150 & \\
\hline${ }^{136} \mathrm{Ba}$ & Stable & 0.000 & 0.001 & 0.002 & Waste \\
\hline${ }^{137} \mathrm{Ba}$ & Stable & 0.000 & 0.006 & 0.045 & Waste \\
\hline${ }^{138} \mathrm{Ba}$ & Stable & 0.004 & 0.050 & 0.149 & Waste \\
\hline${ }^{138} \mathrm{Ba}$ & Stable & 0.002 & 0.002 & 0.002 & Fuel \\
\hline Rh & & 0.005 & 0.055 & 0.164 & \\
\hline${ }^{103} \mathrm{Rh}$ & Stable & 0.005 & 0.055 & 0.164 & Waste \\
\hline Te & & 0.005 & 0.050 & 0.145 & \\
\hline${ }^{99} \mathrm{Tc}$ & $2.1 \times 10^{5} \mathrm{yrs}$ & 0.005 & 0.050 & 0.145 & Waste \\
\hline La & & 0.005 & 0.049 & 0.142 & \\
\hline${ }^{139} \mathrm{La}$ & Stable & 0.005 & 0.049 & 0.142 & Waste \\
\hline Pr & & 0.004 & 0.044 & 0.130 & \\
\hline${ }^{141} \operatorname{Pr}$ & Stable & 0.004 & 0.044 & 0.130 & Waste \\
\hline Sm & & 0.003 & 0.036 & 0.116 & \\
\hline${ }^{147} \mathrm{Sm}$ & $1.1 \times 10^{11} \mathrm{yrs}$ & 0.000 & 0.012 & 0.046 & Waste \\
\hline${ }^{149} \mathrm{Sm}$ & Stable & 0.001 & 0.011 & 0.032 & Waste \\
\hline${ }^{151} \mathrm{Sm}$ & 88.8 yrs & 0.001 & 0.006 & 0.017 & Waste \\
\hline${ }^{152} \mathrm{Sm}$ & Stable & 0.000 & 0.005 & 0.014 & Waste \\
\hline${ }^{154} \mathrm{Sm}$ & Stable & 0.000 & 0.002 & 0.006 & Waste \\
\hline Te & & 0.003 & 0.026 & 0.075 & Fuel \\
\hline${ }^{125} \mathrm{Te}$ & Stable & 0.000 & 0.000 & 0.002 & Waste \\
\hline${ }^{128} \mathrm{Te}$ & $2.2 \times 10^{24} \mathrm{yrs}$ & 0.001 & 0.006 & 0.017 & Waste \\
\hline${ }^{130} \mathrm{Te}$ & $8.2 \times 10^{20} \mathrm{yrs}$ & 0.002 & 0.019 & 0.056 & Waste \\
\hline $\mathbf{S r}$ & & 0.003 & 0.030 & 0.076 & \\
\hline${ }^{88} \mathrm{Sr}$ & Stable & 0.001 & 0.012 & 0.035 & Waste \\
\hline${ }^{90} \mathrm{Sr}$ & 28.9 yrs & 0.001 & 0.017 & 0.041 & Waste \\
\hline${ }^{90} \mathrm{Sr}$ & 28.9 yrs & 0.001 & 0.001 & 0.001 & Fuel \\
\hline I & & 0.001 & 0.010 & 0.027 & \\
\hline${ }^{127} \mathrm{I}$ & Stable & 0.000 & 0.001 & 0.002 & Fuel \\
\hline${ }^{127} \mathrm{I}$ & Stable & 0.000 & 0.000 & 0.001 & Waste \\
\hline${ }^{129} \mathrm{I}$ & $1.6 \times 10^{7} \mathrm{yrs}$ & 0.001 & 0.008 & 0.022 & Fuel \\
\hline${ }^{129} \mathrm{I}$ & $1.6 \times 10^{7} \mathrm{yrs}$ & 0.000 & 0.002 & 0.005 & Waste \\
\hline
\end{tabular}




(n)

